\title{
EFFECT OF SEAWEED EXTRACT ON THE GROWTH AND YIELD OF FABA BEAN (VICIA FABA L.)
}

\author{
Rawheya A. Salah El Din*, Ahmed A. Elbakry**, Safia M. Ghazi** \\ and Olfat M. Abdel Hamid** \\ * Botany and Microbiology Department, Faculty of Science, Al Azhar University \\ (Girl Branch), Egypt. \\ ** Botany and Microbiology Department, Faculty of Science, Helwan University, \\ Egypt
}

\begin{abstract}
The effect of seaweed extracts as a liquid fertilizer on growth, yield and biochemical constituents of faba bean was studied. The seaweed extracts were prepared from Sargassum latifolium, Halimeda opuntia collected from the Red Sea coast and Ulva rigida collected from Mediterranean Sea coast. The algal extracts used as foliar spray in a field experiment enhanced the faba bean growth criteria. Root parameters (root fresh and dry weights, root length number of root nodules, nitrogenase enzyme activity of root) and shoot parameters (shoot fresh and dry weights, shoot length), also the pigment content, number of tillers, number of pods/ plant, grain yield, straw yield and seed index were increased. Some biochemical metablites (total carbohydrates, total protein and also the percentage of N, P and K) of seeds increased by seaweed extract application.
\end{abstract}

Key word: Faba bean, Foliar spray, Seaweed, Red Sea, Mediterranean Coasts

\section{Introduction}

Seaweed extracts are often regarded as soft or natural products that can influence crop growth and development (Norrie and Hiltz, 1999). A wide range of beneficial effects has been observed including increasing crop yield, nutrient uptake, resistance to frost and stress conditions, longer shelf life of fruit, improved seed germination, and reduced incidence of fungal and insect attack and reduced the effect of salinity stress on membrane permeability (Løpez-Mosquera and Pazos, 1997; Malaguti et al., 2002; Hu et al., 2004 and Wang et al., 2005). The effect of crude seaweed extracts of three green seaweeds (Cladophora dalmatica, Enteromorpha intestinalis, Ulva lactuca) and the three red algae (Corallina mediterranea, Jania rubens, Pterocladia pinnate) from the Egyptian Mediterranean Sea coast were studied by El-Sheekh and El-Saied (2000) on seed germination, growth of seedlings, chlorophyll content and other metabolic activities of Vicia faba. They found that the crude extract of $C$. dalmatica showed maximal activity, and it increased seed germination, length of main root and shoot systems and the number of lateral roots. Also, all the crude extracts of seaweed increased protein content in root and shoot systems, total soluble sugars and chlorophyll content in leaves. The cytokinin content of the green algae was higher than that in red algae. Growth of seedlings of $V$. faba was stimulated but to different degrees. Legumes are a major source of protein in human and animal 
nutrition and play a key role in crop rotations in most parts of the world. When it grows in rotation with other crops, under certain environmental conditions, they can improve soil fertility and reduce the incidence of weeds, diseases and pests (Mwanamwenge et al., 1998).

This study was conducted to investigate the influence of field application of seaweed extract (SWE) on some growth and yield criteria of broad bean.

\section{Materials and Methods}

\section{Algal collection}

Halimeda opuntia (L.) Lamour was collected from Marsa Alam (Red Sea coast), Sargassum latifolium (Turner) C. Agardii was collected from Hurgada Red Sea coast in June 2003 and Ulva rigida C. Agardii from Baltim, Mediterranean coast in June 2004. Epiphytes were removed and the samples were successively rinsed with sea water and distilled water. Seaweeds were then air dried and powdered using electric mill.

\section{Plant material}

Faba bean (Vicia faba cv. Giza 3) was obtained from Field Crops Research Institute, Agricultural Research Center (ARC), Giza, Egypt.

\section{Preparation of foliar spray}

Hundred gram of algal powder were extracted by $80 \%$ methanol (the solvent was evaporated under reduced pressure), then the defatted powder was reextracted with hot water for $24 \mathrm{hrs}$, both extracts were mixed together and completed to one liter with distilled water to obtain a solution of $10 \%$ concentration/dry weight used as a stock solution from which the different concentrations was prepared by dilution and used as foliar spray.

\section{Treatments and experimental design}

A field experiment was carried out during (November-April) 2004/2005 at Ismaillia Experimental Farm Research Station (ARC). Plot area (6 rows) was 1 $\mathrm{m}^{2}$ and the experimental design used was arranged in a complete randomized design with three replicates for each treatment. Plots received the dose of N P K recommended by the Egyptian Agricultural Ministry.

Faba bean seeds were drilled in rows with a distance of $60 \mathrm{~cm}$ apart at a rate of ( $60 \mathrm{~kg}$ seeds/fed). The type of irrigation of soil was sprinkle irrigation. The algal extract was applied as 3 doses: 15 days $-250 \mathrm{~mL}$ of extract/plot, 45 days $500 \mathrm{~mL}$ of extract/plot, and 75 days $-750 \mathrm{~mL}$ of extract/plot. Five different concentrations were used $(0.2,0.4,0.6,0.8$ and $1 \% \mathrm{v}$ : v) in addition to control (water). Each treatment was applied to 3 plots. Plant samples were collected at three intervals, the first (stage A) after 45 days from sowing and before the second spraying, the second after 75 days (stage B) and the last one after 120 days (stage C). Three plants were taken randomly from each plot at each stage to determine Growth and yield parameters. 


\section{Determination of Metabolites content of Vicia faba}

The total nitrogen was determined using microKjeldahl method (Pirie, 1955). The crude protein was calculated by multiplying total nitrogen per cent in both straw and seeds by the factor 6.25. The total phosphorus was determined colorimetrically using the phosphomolybdate method (Woods and Mellon, 1965). The total potassium was determined using flame photometer method (Williams and Twine, 1960). The total protein was determined by spectrophotometric method (Lowery et al. 1951), total carbohydrates colorimetrically determination as describe by Dubois et al. (1956). Pigments determinations were done according to the method of Metzner et al. (1965).

\section{Statistical Analysis}

Results were statistically analyzed by calculating the analysis of variance, in completely randomized design (Snedecor and Cochran 1981) and means were compared by multiple range test (Duncan 1955).

\section{Results}

Data in Table (1) indicated that the overall root growth of faba bean were improved by seaweed concentrated foliar application. On the other hand, foliar application had no significant effect on shoot growth parameter (Table 2).

Number of nodules increased by foliar seaweed application as compared with the control during the growth stages. It appeared that the concentration $(0.8 \%)$ has the best number of nodules for all growth stages A, B and C (Table 3). Also, data in Table (3) showed that activity of nitrogenase enzyme in root nodules had increased due to seaweed application as foliar spray.

Significant increases in Chlorophyll a, b and carotenoids content were obtained; but there were no steady increase with increasing concentrations of seaweed application (Table 4).

Data in Table (5) Showed that foliar sprays improved the concentration level of NPK in faba bean especially at $0.6 \%$ concentration. Also, from the same table it shows that the three studied species have positive impact on the NPK levels in side the faba bean.

Data in Table (6) noted that the tiller number was positively affected by the foliar spray in all concentrations and with all three studied algal species. While, the pods number were positively affected only at $0.6 \%$ concentration and $H$. opuntia species recorded better pod number than that of the two other studied species.

Result presented in Figure (1) showed that the foliar spray increased significantly the yields of seed index as well as the grain yield specialy at concentration $0.6 \%$. On the other hand, total seed carbohydrate and protein 
Table (1): Root length, fresh and dry weights of faba bean foliar spray with different concentrations (\% w: v) of three algal species extracts through growth stages of plant: A (45 days), B (75 days) and C (120 days). Data expressed as a mean of three replicates.

\begin{tabular}{|c|c|c|c|c|c|c|c|c|c|c|c|c|}
\hline \multirow{3}{*}{ 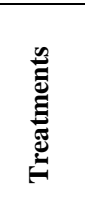 } & \multicolumn{12}{|c|}{ Root fresh weight (g) } \\
\hline & \multicolumn{4}{|c|}{ Stage A } & \multicolumn{4}{|c|}{ Stage B } & \multicolumn{4}{|c|}{ Stage $\mathrm{C}$} \\
\hline & 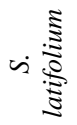 & 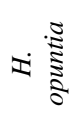 & 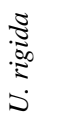 & $\stackrel{\Xi}{\stackrel{\Xi}{\Sigma}}$ & 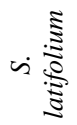 & 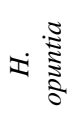 & $\begin{array}{l}\mathbb{3} \\
: 0 \\
: 0 \\
0\end{array}$ & $\stackrel{\Xi}{\Xi}$ & 的莣 & 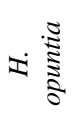 & 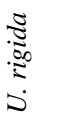 & $\sum_{\Sigma}^{\Xi}$ \\
\hline Cont. & $\begin{array}{c}4.9 \\
\mathrm{~g}\end{array}$ & $\begin{array}{c}4.9 \\
\mathrm{~g}\end{array}$ & $\begin{array}{c}4.9 \\
\mathrm{~g}\end{array}$ & $\begin{array}{c}4.90 \\
d\end{array}$ & $\begin{array}{r}7.0 \\
\mathrm{~cd}\end{array}$ & $\begin{array}{l}7.0 \\
\mathrm{~cd}\end{array}$ & $\begin{array}{l}7.0 \\
\mathrm{~cd}\end{array}$ & $\begin{array}{l}7.0 \\
\text { cd }\end{array}$ & $\begin{array}{l}6.8 \\
\text { de }\end{array}$ & $\begin{array}{l}6.8 \\
\text { de }\end{array}$ & $\begin{array}{c}6.8 \\
\text { de }\end{array}$ & $\begin{array}{c}6.8 \\
\text { c }\end{array}$ \\
\hline $0.2 \%$ & $\begin{array}{c}5.14 \\
\text { fg }\end{array}$ & $\begin{array}{l}6.2 \\
\mathrm{~cd}\end{array}$ & $\begin{array}{l}6.46 \\
\text { bcd }\end{array}$ & $\begin{array}{c}5.93 \\
\text { b }\end{array}$ & $\begin{array}{c}5.3 \\
\mathrm{~g}\end{array}$ & $\begin{array}{c}8.0 \\
a\end{array}$ & $\begin{array}{l}6.6 \\
\mathrm{de}\end{array}$ & $\begin{array}{c}6.63 \\
\text { de }\end{array}$ & $\begin{array}{c}6.06 \\
\text { ef }\end{array}$ & $\begin{array}{l}7.5 \\
\mathrm{~cd}\end{array}$ & $\begin{array}{c}9.0 \\
\mathrm{a}\end{array}$ & $\begin{array}{c}7.52 \\
\text { b }\end{array}$ \\
\hline $0.4 \%$ & $\begin{array}{c}6.97 \\
\mathrm{ab}\end{array}$ & $\begin{array}{c}6.34 \\
\mathrm{~cd}\end{array}$ & $\begin{array}{c}7.25 \\
\mathrm{a}\end{array}$ & $\begin{array}{c}6.85 \\
\text { a }\end{array}$ & $\begin{array}{c}5.5 \\
\mathrm{~g}\end{array}$ & $\begin{array}{c}5.5 \\
\mathrm{~g}\end{array}$ & $\begin{array}{l}5.8 \\
\text { fg }\end{array}$ & $\begin{array}{c}5.60 \\
c\end{array}$ & $\begin{array}{c}8.91 \\
\text { ab }\end{array}$ & $\begin{array}{l}8.28 \\
a b c\end{array}$ & $\begin{array}{l}8.52 \\
\text { abc }\end{array}$ & $\begin{array}{c}8.57 \\
\text { a }\end{array}$ \\
\hline $0.6 \%$ & $\begin{array}{c}4.57 \\
\mathrm{~g}\end{array}$ & $\begin{array}{c}5.59 \\
\text { ef }\end{array}$ & $\begin{array}{c}6.18 \\
\mathrm{~cd}\end{array}$ & $\begin{array}{c}5.45 \\
\text { e }\end{array}$ & $\begin{array}{l}6.1 \\
\text { ef }\end{array}$ & $\begin{array}{c}8.1 \\
a\end{array}$ & $\begin{array}{l}7.6 \\
a b\end{array}$ & $\begin{array}{c}7.26 \\
7 \mathrm{a}\end{array}$ & $\begin{array}{l}7.80 \\
\text { bcd }\end{array}$ & $\begin{array}{c}6.73 \\
\text { de }\end{array}$ & $\begin{array}{l}7.78 \\
\text { bcd }\end{array}$ & $\begin{array}{c}7.43 \\
7 \mathrm{~b}\end{array}$ \\
\hline $0.8 \%$ & $\begin{array}{c}4.94 \\
\mathrm{~g}\end{array}$ & $\begin{array}{l}6.69 \\
\mathrm{abc}\end{array}$ & $\begin{array}{c}6.19 \\
\mathrm{~cd}\end{array}$ & $\begin{array}{c}5.94 \\
\text { b }\end{array}$ & $\begin{array}{l}7.7 \\
a b\end{array}$ & $\begin{array}{c}7.55 \\
a b\end{array}$ & $\begin{array}{c}4.7 \\
\mathrm{~h}\end{array}$ & $\begin{array}{c}6.65 \\
\text { de }\end{array}$ & $\begin{array}{c}5.73 \\
\text { ef }\end{array}$ & $\begin{array}{c}5.3 \\
\mathrm{f}\end{array}$ & $\begin{array}{c}8.86 \\
\mathrm{ab}\end{array}$ & $\begin{array}{c}6.63 \\
0 \mathrm{c}\end{array}$ \\
\hline $1 \%$ & $\begin{array}{l}6.7 \\
a b c\end{array}$ & $\begin{array}{c}3.88 \\
\text { h }\end{array}$ & $\begin{array}{l}6.0 \\
\mathrm{de}\end{array}$ & $\begin{array}{c}5.53 \\
\text { c }\end{array}$ & $\begin{array}{c}8.0 \\
\mathrm{a}\end{array}$ & $\begin{array}{c}6.70 \\
\mathrm{~cd}\end{array}$ & $\begin{array}{c}7.20 \\
b c\end{array}$ & $\begin{array}{c}7.30 \\
\mathrm{a}\end{array}$ & $\begin{array}{c}6.86 \\
\text { de }\end{array}$ & $\begin{array}{l}8.60 \\
a b c\end{array}$ & $\begin{array}{l}8.24 \\
\text { abc }\end{array}$ & $\begin{array}{c}7.9 \\
\text { b }\end{array}$ \\
\hline Mean & $\begin{array}{c}\mathbf{5 . 5 4} \\
\text { b }\end{array}$ & $\begin{array}{c}\mathbf{5 . 6 0} \\
\text { b }\end{array}$ & $\begin{array}{c}6.16 \\
a\end{array}$ & & $\begin{array}{c}6.6 \\
b\end{array}$ & $\begin{array}{c}7.14 \\
\mathrm{a}\end{array}$ & $\begin{array}{c}6.48 \\
b\end{array}$ & & $\begin{array}{c}7.03 \\
\text { b }\end{array}$ & $\begin{array}{c}7.2 \\
\text { b }\end{array}$ & $\begin{array}{c}8.2 \\
\mathrm{a}\end{array}$ & \\
\hline \multicolumn{13}{|c|}{ Root dry weight (g) } \\
\hline Cont. & $\begin{array}{c}0.97 \\
\mathrm{~d}\end{array}$ & $\begin{array}{c}0.97 \\
\mathrm{~d}\end{array}$ & $\begin{array}{c}0.97 \\
\mathrm{~d}\end{array}$ & $\begin{array}{c}0.97 \\
\text { c }\end{array}$ & $\begin{array}{c}2.10 \\
\mathrm{~cd}\end{array}$ & $\begin{array}{c}2.10 \\
\mathrm{~cd}\end{array}$ & $\begin{array}{c}2.10 \\
\mathrm{~cd}\end{array}$ & $\begin{array}{c}2.10 \\
\text { cd }\end{array}$ & $\begin{array}{c}2.05 \\
\mathrm{~cd}\end{array}$ & $\begin{array}{c}2.05 \\
\mathrm{~cd}\end{array}$ & $\begin{array}{c}2.05 \\
\mathrm{~cd}\end{array}$ & $\begin{array}{c}2.05 \\
\mathrm{~b}\end{array}$ \\
\hline $0.2 \%$ & $\begin{array}{c}0.96 \\
d\end{array}$ & $\begin{array}{l}1.22 \\
\text { bcd }\end{array}$ & $\begin{array}{c}1.62 \\
\mathrm{a}\end{array}$ & $\begin{array}{c}1.27 \\
\text { ab }\end{array}$ & $\begin{array}{c}1.56 \\
\mathrm{f}\end{array}$ & $\begin{array}{c}2.10 \\
\mathrm{~cd}\end{array}$ & $\begin{array}{c}2.10 \\
\mathrm{~cd}\end{array}$ & $\begin{array}{c}1.92 \\
\text { c }\end{array}$ & $\begin{array}{c}1.71 \\
\mathrm{e}\end{array}$ & $\begin{array}{c}2.05 \\
\mathrm{~cd}\end{array}$ & $\begin{array}{c}2.27 \\
b\end{array}$ & $\begin{array}{c}2.01 \\
b\end{array}$ \\
\hline $0.4 \%$ & $\begin{array}{c}1.39 \\
\mathrm{ab}\end{array}$ & $\begin{array}{c}1.58 \\
\mathrm{a}\end{array}$ & $\begin{array}{l}1.23 \\
\mathrm{bcd}\end{array}$ & $\begin{array}{c}1.40 \\
\mathrm{a}\end{array}$ & $\begin{array}{c}2.30 \\
b c\end{array}$ & $\begin{array}{c}1.88 \\
\mathrm{de}\end{array}$ & $\begin{array}{c}2.48 \\
\mathrm{ab}\end{array}$ & $\begin{array}{c}2.22 \\
\text { ab }\end{array}$ & $\begin{array}{l}2.13 \\
\text { bcd }\end{array}$ & $\begin{array}{c}2.05 \\
\mathrm{~cd}\end{array}$ & $\begin{array}{c}2.56 \\
\mathrm{a}\end{array}$ & $\begin{array}{c}2.25 \\
\mathrm{a}\end{array}$ \\
\hline $0.6 \%$ & $\begin{array}{l}1.25 \\
\mathrm{bcd}\end{array}$ & $\begin{array}{l}1.28 \\
\text { bcd }\end{array}$ & $\begin{array}{l}1.19 \\
\text { bcd }\end{array}$ & $\begin{array}{c}1.24 \\
\text { b }\end{array}$ & $\begin{array}{c}1.73 \\
\text { ef }\end{array}$ & $\begin{array}{c}2.60 \\
\mathrm{a}\end{array}$ & $\begin{array}{c}1.89 \\
\mathrm{de}\end{array}$ & $\begin{array}{c}2.07 \\
\text { b }\end{array}$ & $\begin{array}{c}1.49 \\
\text { fg }\end{array}$ & $\begin{array}{c}2.17 \\
b c\end{array}$ & $\begin{array}{c}1.99 \\
\mathrm{~cd}\end{array}$ & $\begin{array}{c}1.88 \\
\mathrm{c}\end{array}$ \\
\hline $0.8 \%$ & $\begin{array}{c}1.03 \\
\mathrm{~cd}\end{array}$ & $\begin{array}{r}1.0 \\
\mathrm{~cd}\end{array}$ & $\begin{array}{c}1.02 \\
\mathrm{~cd}\end{array}$ & $\begin{array}{c}1.02 \\
\text { c }\end{array}$ & $\begin{array}{c}2.40 \\
\mathrm{ab}\end{array}$ & $\begin{array}{c}2.41 \\
a b\end{array}$ & $\begin{array}{c}1.66 \\
\text { ef }\end{array}$ & $\begin{array}{c}2.16 \\
\text { ab }\end{array}$ & $\begin{array}{c}1.63 \\
\text { ef }\end{array}$ & $\begin{array}{l}2.09 \\
\text { bcd }\end{array}$ & $\begin{array}{c}1.96 \\
d\end{array}$ & $\begin{array}{c}1.89 \\
\mathrm{c}\end{array}$ \\
\hline $1 \%$ & $\begin{array}{c}1.07 \\
\mathrm{~cd}\end{array}$ & $\begin{array}{c}1.59 \\
\mathrm{a}\end{array}$ & $\begin{array}{l}1.18 \\
\text { bcd }\end{array}$ & $\begin{array}{c}1.28 \\
\text { ab }\end{array}$ & $\begin{array}{c}2.10 \\
\mathrm{~cd}\end{array}$ & $\begin{array}{l}2.36 \\
a b c\end{array}$ & $\begin{array}{c}2.30 \\
b c\end{array}$ & $\begin{array}{c}2.25 \\
\mathrm{a}\end{array}$ & $\begin{array}{c}2.25 \\
b\end{array}$ & $\begin{array}{c}1.40 \\
\mathrm{~g}\end{array}$ & $\begin{array}{c}2.00 \\
\mathrm{~cd}\end{array}$ & $\begin{array}{c}1.88 \\
\mathrm{c}\end{array}$ \\
\hline Mean & $\begin{array}{c}1.11 \\
b\end{array}$ & $\begin{array}{c}1.27 \\
\mathrm{a}\end{array}$ & $\begin{array}{c}1.20 \\
\text { ab }\end{array}$ & & $\begin{array}{c}2.03 \\
\text { b }\end{array}$ & $\begin{array}{c}2.24 \\
\text { a }\end{array}$ & $\begin{array}{c}2.09 \\
\text { b }\end{array}$ & & $\begin{array}{c}1.87 \\
\text { c }\end{array}$ & $\begin{array}{c}1.96 \\
b\end{array}$ & $\begin{array}{c}2.13 \\
\text { a }\end{array}$ & \\
\hline & & & & & Roo & ength & & & & & & \\
\hline Cont. & $\begin{array}{c}15.0 \\
\mathrm{~cd}\end{array}$ & $\begin{array}{c}15.0 \\
\mathrm{~cd}\end{array}$ & $\begin{array}{c}15.0 \\
\mathrm{~cd}\end{array}$ & $\begin{array}{c}15.0 \\
\text { b }\end{array}$ & $\begin{array}{c}17.4 \\
\mathrm{ab}\end{array}$ & $\begin{array}{c}17.4 \\
\mathrm{ab}\end{array}$ & $\begin{array}{c}17.4 \\
\mathrm{ab}\end{array}$ & $\begin{array}{c}17.4 \\
\text { ab }\end{array}$ & $\begin{array}{c}17.2 \\
\mathrm{~cd}\end{array}$ & $\begin{array}{c}17.2 \\
\mathrm{~cd}\end{array}$ & $\begin{array}{c}17.2 \\
\mathrm{~cd}\end{array}$ & $\begin{array}{c}17.2 \\
\mathrm{~cd}\end{array}$ \\
\hline $0.2 \%$ & $\begin{array}{c}14.8 \\
\mathrm{de}\end{array}$ & $\begin{array}{c}12.1 \\
\mathrm{j}\end{array}$ & $\begin{array}{c}14.2 \\
\mathrm{~g}\end{array}$ & $\begin{array}{c}13.7 \\
\text { d }\end{array}$ & $\begin{array}{l}16.5 \\
\text { abcd }\end{array}$ & $\begin{array}{l}16.2 \\
\text { bcde }\end{array}$ & $\begin{array}{l}16.7 \\
\text { abcd }\end{array}$ & $\begin{array}{c}16.5 \\
\text { bc }\end{array}$ & $\begin{array}{c}17.8 \\
\text { bc }\end{array}$ & $\begin{array}{l}17.5 \\
\text { bcd }\end{array}$ & $\begin{array}{c}16.0 \\
\mathrm{f}\end{array}$ & $\begin{array}{c}17.1 \\
b\end{array}$ \\
\hline $0.4 \%$ & $\begin{array}{c}13.2 \\
h\end{array}$ & $\begin{array}{c}12.3 \\
\mathrm{ij}\end{array}$ & $\begin{array}{c}14.5 \\
\text { efg }\end{array}$ & $\begin{array}{c}13.3 \\
\text { e }\end{array}$ & $\begin{array}{l}17.3 \\
a b c\end{array}$ & $\begin{array}{c}17.8 \\
\mathrm{ab}\end{array}$ & $\begin{array}{c}17.8 \\
\mathrm{ab}\end{array}$ & $\begin{array}{c}17.7 \\
\text { a }\end{array}$ & $\begin{array}{c}17.6 \\
\text { bc }\end{array}$ & $\begin{array}{l}17.5 \\
\text { bcd }\end{array}$ & $\begin{array}{c}18.1 \\
\mathrm{~b}\end{array}$ & $\begin{array}{c}17.7 \\
\mathrm{a}\end{array}$ \\
\hline $0.6 \%$ & $\begin{array}{c}14.3 \\
\text { fg }\end{array}$ & $\begin{array}{c}16.8 \\
\mathrm{a}\end{array}$ & $\begin{array}{c}16.5 \\
\mathrm{a}\end{array}$ & $\begin{array}{c}15.8 \\
\text { a }\end{array}$ & $\begin{array}{c}16.7 \\
\text { abcd }\end{array}$ & $\begin{array}{c}17.3 \\
a b\end{array}$ & $\begin{array}{c}16.8 \\
\text { abcd }\end{array}$ & $\begin{array}{l}17.0 \\
\text { abc }\end{array}$ & $\begin{array}{c}15.3 \\
\mathrm{~g}\end{array}$ & $\begin{array}{c}17.7 \\
b c\end{array}$ & $\begin{array}{c}18.1 \\
\mathrm{~b}\end{array}$ & $\begin{array}{c}17.0 \\
b\end{array}$ \\
\hline $0.8 \%$ & $\begin{array}{c}15.3 \\
\text { bc }\end{array}$ & $\begin{array}{c}15.3 \\
b c\end{array}$ & $\begin{array}{c}12.7 \\
\mathrm{i}\end{array}$ & $\begin{array}{c}14.4 \\
\text { c }\end{array}$ & $\begin{array}{l}16.2 \\
\text { bcde }\end{array}$ & $\begin{array}{c}17.3 \\
a b\end{array}$ & $\begin{array}{c}15.2 \\
\mathrm{de}\end{array}$ & $\begin{array}{c}16.2 \\
\text { c }\end{array}$ & $\begin{array}{c}16.5 \\
\text { ef }\end{array}$ & $\begin{array}{c}17.8 \\
\mathrm{bc}\end{array}$ & $\begin{array}{c}15.1 \\
\mathrm{~g}\end{array}$ & $\begin{array}{c}16.5 \\
\mathrm{c}\end{array}$ \\
\hline $1 \%$ & $\begin{array}{c}14.8 \\
\mathrm{de}\end{array}$ & $\begin{array}{c}14.7 \\
\text { def }\end{array}$ & $\begin{array}{c}15.7 \\
\mathrm{~b}\end{array}$ & $\begin{array}{c}15.0 \\
\text { b }\end{array}$ & $\begin{array}{c}15.5 \\
d\end{array}$ & $\begin{array}{c}18.3 \\
a b\end{array}$ & $\begin{array}{c}14.5 \\
\mathrm{c}\end{array}$ & $\begin{array}{c}16.1 \\
\text { c }\end{array}$ & $\begin{array}{l}17.5 \\
\text { bcd }\end{array}$ & $\begin{array}{c}19.6 \\
\mathrm{a}\end{array}$ & $\begin{array}{c}16.8 \\
\mathrm{de}\end{array}$ & $\begin{array}{c}17.9 \\
\mathrm{a}\end{array}$ \\
\hline Mean & $\begin{array}{c}14.5 \\
\mathrm{a}\end{array}$ & $\begin{array}{c}14.3 \\
\text { c }\end{array}$ & $\begin{array}{c}14.7 \\
\mathrm{a}\end{array}$ & & $\begin{array}{c}16.6 \\
\text { b }\end{array}$ & $\begin{array}{c}17.4 \\
\mathrm{a}\end{array}$ & $\begin{array}{c}16.4 \\
\text { b }\end{array}$ & & $\begin{array}{c}17.0 \\
\text { b }\end{array}$ & $\begin{array}{c}17.9 \\
\text { a }\end{array}$ & $\begin{array}{c}16.9 \\
\text { b }\end{array}$ & \\
\hline
\end{tabular}

Different letters are significantly different at $p<0.05$. 
Table (2): Shoot length, fresh and dry weights of faba bean foliar spray with different concentrations (\% w: v) of three algal species extracts through growth stages of plant: A (45 days), B (75 days) and C (120 days). Data expressed as a mean of three replicates.

\begin{tabular}{|c|c|c|c|c|c|c|c|c|c|c|c|c|}
\hline \multirow{3}{*}{ 䔸 } & \multicolumn{12}{|c|}{ Shoot fresh weight (g) } \\
\hline & \multicolumn{4}{|c|}{ Stage A } & \multicolumn{4}{|c|}{ Stage B } & \multicolumn{4}{|c|}{ Stage C } \\
\hline & 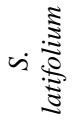 & 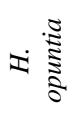 & 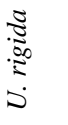 & $\stackrel{\Xi}{\tilde{\Xi}}$ & 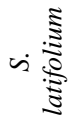 & 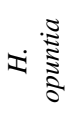 & $\begin{array}{l}3 \\
0 \\
0 \\
0 \\
0 \\
0 \\
0\end{array}$ & 胥 & 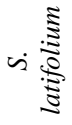 & ¿i & 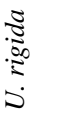 & 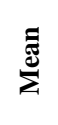 \\
\hline Cont & $\begin{array}{l}10.8 \\
\text { bcd }\end{array}$ & $\begin{array}{l}10.8 \\
\text { bcd }\end{array}$ & $\begin{array}{l}10.8 \\
\text { bcd }\end{array}$ & $\begin{array}{c}10.8 \\
\text { b }\end{array}$ & $\begin{array}{c}27.5 \\
\mathrm{c}\end{array}$ & $\begin{array}{c}29.0 \\
\mathrm{c}\end{array}$ & $\begin{array}{c}29.0 \\
\mathrm{c} \\
\end{array}$ & $\begin{array}{c}28.5 \\
\text { b }\end{array}$ & $\begin{array}{c}44.4 \\
\text { fg }\end{array}$ & $\begin{array}{c}44.4 \\
\mathrm{fg}\end{array}$ & $\begin{array}{c}44.4 \\
\text { fg } \\
\end{array}$ & $\begin{array}{c}44.4 \\
\text { c }\end{array}$ \\
\hline $0.2 \%$ & $\begin{array}{l}9.8 \\
\text { def }\end{array}$ & $\begin{array}{l}9.62 \\
\text { efg }\end{array}$ & $\begin{array}{l}10.5 \\
\text { bcde }\end{array}$ & $\begin{array}{c}9.98 \\
\text { c }\end{array}$ & $\begin{array}{l}25.1 \\
\text { efg }\end{array}$ & $\begin{array}{l}26.8 \\
\text { cde }\end{array}$ & $\begin{array}{c}25.8 \\
\text { def }\end{array}$ & $\begin{array}{c}25.9 \\
\text { de }\end{array}$ & $\begin{array}{c}35.8 \\
\mathrm{j}\end{array}$ & $\begin{array}{c}39.3 \\
\text { hi }\end{array}$ & $\begin{array}{c}39.2 \\
\text { hi }\end{array}$ & $\begin{array}{c}38.1 \\
\text { d }\end{array}$ \\
\hline $0.4 \%$ & $\begin{array}{c}12.2 \\
\mathrm{a} \\
\end{array}$ & $\begin{array}{c}11.5 \\
\mathrm{ab}\end{array}$ & $\begin{array}{l}10.8 \\
\text { bcd } \\
\end{array}$ & $\begin{array}{c}11.5 \\
\mathrm{a} \\
\end{array}$ & $\begin{array}{c}23.5 \\
\mathrm{gh}\end{array}$ & $\begin{array}{c}32.0 \\
b\end{array}$ & $\begin{array}{c}24.9 \\
\mathrm{fg} \\
\end{array}$ & $\begin{array}{c}26.8 \\
\text { cd }\end{array}$ & $\begin{array}{c}41.4 \\
\text { gh }\end{array}$ & $\begin{array}{c}52.8 \\
\mathrm{~d} \\
\end{array}$ & $\begin{array}{c}52.6 \\
\mathrm{~d} \\
\end{array}$ & $\begin{array}{c}48.9 \\
b\end{array}$ \\
\hline $0.6 \%$ & $\begin{array}{c}11.2 \\
b c\end{array}$ & $\begin{array}{c}9.00 \\
\text { gh }\end{array}$ & $\begin{array}{l}9.61 \\
\text { efg }\end{array}$ & $\begin{array}{c}9.94 \\
\text { c }\end{array}$ & $\begin{array}{c}26.1 \\
\text { def }\end{array}$ & $\begin{array}{c}35.5 \\
\mathrm{a}\end{array}$ & $\begin{array}{c}28.6 \\
c\end{array}$ & $\begin{array}{c}30.0 \\
\text { a }\end{array}$ & $\begin{array}{c}36.0 \\
\mathrm{j}\end{array}$ & $\begin{array}{c}69.8 \\
\mathrm{a}\end{array}$ & $\begin{array}{c}65.4 \\
\mathrm{~b}\end{array}$ & $\begin{array}{c}57.0 \\
\text { a }\end{array}$ \\
\hline $0.8 \%$ & $\begin{array}{c}9.17 \\
\text { gh }\end{array}$ & $\begin{array}{l}9.77 \\
\text { defg }\end{array}$ & $\begin{array}{c}9.38 \\
\text { fg }\end{array}$ & $\begin{array}{c}9.44 \\
\text { c }\end{array}$ & $\begin{array}{c}27.4 \\
\mathrm{~cd}\end{array}$ & $\begin{array}{l}25.0 \\
\text { efg }\end{array}$ & $\begin{array}{c}22.6 \\
\text { hi }\end{array}$ & $\begin{array}{c}25.0 \\
\text { e }\end{array}$ & $\begin{array}{c}48.0 \\
\mathrm{e}\end{array}$ & $\begin{array}{c}37.9 \\
\mathrm{ij}\end{array}$ & $\begin{array}{c}44.3 \\
\mathrm{fg}\end{array}$ & $\begin{array}{c}43.4 \\
3 \mathrm{c}\end{array}$ \\
\hline $1 \%$ & $\begin{array}{c}11.1 \\
\mathrm{bc}\end{array}$ & $\begin{array}{l}10.3 \\
\text { cdef }\end{array}$ & $\begin{array}{c}8.24 \\
\mathrm{~h}\end{array}$ & $\begin{array}{c}9.91 \\
\text { c }\end{array}$ & $\begin{array}{c}32.9 \\
\mathrm{~b}\end{array}$ & $\begin{array}{c}21.5 \\
\text { i }\end{array}$ & $\begin{array}{c}27.2 \\
\mathrm{~cd}\end{array}$ & $\begin{array}{c}27.2 \\
\text { bc }\end{array}$ & $\begin{array}{c}46.3 \\
\text { ef }\end{array}$ & $\begin{array}{c}35.8 \\
\mathrm{j}\end{array}$ & $\begin{array}{c}61.0 \\
\mathrm{c}\end{array}$ & $\begin{array}{c}47.7 \\
\text { b }\end{array}$ \\
\hline Mean & $\begin{array}{c}10.7 \\
\text { a }\end{array}$ & $\begin{array}{c}10.1 \\
\text { b }\end{array}$ & $\begin{array}{c}9.89 \\
\text { b }\end{array}$ & & $\begin{array}{c}27.1 \\
\text { b }\end{array}$ & $\begin{array}{c}28.1 \\
\text { a }\end{array}$ & $\begin{array}{c}26.1 \\
\text { c }\end{array}$ & & $\begin{array}{c}42.0 \\
c\end{array}$ & $\begin{array}{c}46.7 \\
\text { b }\end{array}$ & $\begin{array}{c}51.2 \\
\mathrm{a}\end{array}$ & \\
\hline \multicolumn{13}{|c|}{ Shoot dry weight (g) } \\
\hline Cont & $\begin{array}{c}1.21 \\
b\end{array}$ & $\begin{array}{c}1.21 \\
b\end{array}$ & $\begin{array}{c}1.21 \\
\mathrm{~b}\end{array}$ & $\begin{array}{c}1.21 \\
\text { b }\end{array}$ & $\begin{array}{l}3.9 \\
\text { efg }\end{array}$ & $\begin{array}{l}3.9 \\
\text { efg }\end{array}$ & $\begin{array}{l}3.9 \\
\text { efg }\end{array}$ & $\begin{array}{c}3.90 \\
\text { a }\end{array}$ & $\begin{array}{c}18.0 \\
\mathrm{de}\end{array}$ & $\begin{array}{c}18.0 \\
\mathrm{de}\end{array}$ & $\begin{array}{c}18.0 \\
\mathrm{de}\end{array}$ & $\begin{array}{c}18.0 \\
\text { b }\end{array}$ \\
\hline $0.2 \%$ & $\begin{array}{l}1.06 \\
\text { bcd }\end{array}$ & $\begin{array}{c}0.79 \\
\mathrm{e}\end{array}$ & $\begin{array}{l}1.07 \\
\text { bcd }\end{array}$ & $\begin{array}{c}0.79 \\
\text { c } \\
\end{array}$ & $\begin{array}{c}3.8 \\
\text { fg }\end{array}$ & $\begin{array}{c}4.26 \\
\mathrm{~cd}\end{array}$ & $\begin{array}{c}1.3 \\
\mathrm{k}\end{array}$ & $\begin{array}{c}3.12 \\
\text { c }\end{array}$ & $\begin{array}{c}13.2 \\
\mathrm{i}\end{array}$ & $\begin{array}{c}15.0 \\
\mathrm{j}\end{array}$ & $\begin{array}{c}16.8 \\
\mathrm{hi}\end{array}$ & $\begin{array}{c}15.0 \\
\mathrm{e}\end{array}$ \\
\hline $0.4 \%$ & $\begin{array}{c}1.66 \\
\mathrm{a}\end{array}$ & $\begin{array}{l}1.13 \\
\text { bcd }\end{array}$ & $\begin{array}{l}1.04 \\
\text { bcd }\end{array}$ & $\begin{array}{c}1.28 \\
\mathrm{a}\end{array}$ & $\begin{array}{l}.0 \\
\text { def }\end{array}$ & $\begin{array}{c}2.8 \\
\mathrm{~h}\end{array}$ & $\begin{array}{c}1.9 \\
\mathrm{j}\end{array}$ & $\begin{array}{c}2.90 \\
d\end{array}$ & $\begin{array}{l}14.9 \\
4 \mathrm{jk}\end{array}$ & $\begin{array}{l}16.5 \\
6 \mathrm{hi}\end{array}$ & $\begin{array}{l}17.7 \\
\text { def }\end{array}$ & $\begin{array}{c}16.4 \\
0 \mathrm{~d}\end{array}$ \\
\hline $0.6 \%$ & $\begin{array}{l}1.03 \\
\text { bcd }\end{array}$ & $\begin{array}{l}1.0 \\
\text { bcd }\end{array}$ & $\begin{array}{l}1.14 \\
\text { bcd }\end{array}$ & $\begin{array}{c}1.06 \\
\text { bc }\end{array}$ & $\begin{array}{c}2.97 \\
\mathrm{~h}\end{array}$ & $\begin{array}{l}3.8 \\
\mathrm{fg}\end{array}$ & $\begin{array}{c}4.8 \\
\mathrm{a}\end{array}$ & $\begin{array}{c}3.86 \\
\mathrm{a}\end{array}$ & $\begin{array}{c}17.0 \\
\text { gh }\end{array}$ & $\begin{array}{c}22.8 \\
4 \mathrm{a} \\
\end{array}$ & $\begin{array}{c}21.8 \\
\mathrm{~b}\end{array}$ & $\begin{array}{c}20.5 \\
8 \text { a }\end{array}$ \\
\hline $0.8 \%$ & $\begin{array}{c}0.93 \\
\text { de }\end{array}$ & $\begin{array}{l}0.98 \\
\text { cde }\end{array}$ & $\begin{array}{l}1.0 \\
\text { bcd }\end{array}$ & $\begin{array}{c}0.97 \\
\text { c }\end{array}$ & $\begin{array}{c}2.9 \\
\mathrm{~h}\end{array}$ & $\begin{array}{c}4.36 \\
b c\end{array}$ & $\begin{array}{c}4.6 \\
\mathrm{~g}\end{array}$ & $\begin{array}{c}3.95 \\
\text { a }\end{array}$ & $\begin{array}{c}19.0 \\
\mathrm{c}\end{array}$ & $\begin{array}{l}17.6 \\
\text { efg }\end{array}$ & $\begin{array}{c}14.3 \\
\mathrm{k}\end{array}$ & $\begin{array}{c}17.0 \\
\text { c }\end{array}$ \\
\hline $1 \%$ & $\begin{array}{l}1.07 \\
\text { bcd }\end{array}$ & $\begin{array}{c}1.17 \\
b c\end{array}$ & $\begin{array}{l}1.05 \\
\text { bcd }\end{array}$ & $\begin{array}{c}1.10 \\
b\end{array}$ & $\begin{array}{l}4.19 \\
\text { cde }\end{array}$ & $\begin{array}{c}2.3 \\
\mathrm{i}\end{array}$ & $\begin{array}{c}3.6 \\
\mathrm{~g}\end{array}$ & $\begin{array}{c}3.36 \\
\text { b }\end{array}$ & $\begin{array}{l}17.1 \\
\text { fgh }\end{array}$ & $\begin{array}{c}16.2 \\
\mathrm{i}\end{array}$ & $\begin{array}{c}18.4 \\
d\end{array}$ & $\begin{array}{c}17.2 \\
\text { c }\end{array}$ \\
\hline Mean & $\begin{array}{c}1.16 \\
a\end{array}$ & $\begin{array}{c}1.05 \\
b\end{array}$ & $\begin{array}{c}1.08 \\
b\end{array}$ & & $\begin{array}{c}3.63 \\
\text { a }\end{array}$ & $\begin{array}{c}3.57 \\
\text { a }\end{array}$ & $\begin{array}{c}3.35 \\
\text { b }\end{array}$ & & $\begin{array}{c}16.5 \\
\text { b }\end{array}$ & $\begin{array}{c}18.4 \\
\text { a }\end{array}$ & $\begin{array}{c}17.8 \\
\text { a }\end{array}$ & \\
\hline & & & & & Shoo & length & m) & & & & & \\
\hline Cont & $\begin{array}{c}14.2 \\
\mathrm{~cd}\end{array}$ & $\begin{array}{c}14.2 \\
\mathrm{~cd}\end{array}$ & $\begin{array}{c}14.2 \\
\mathrm{~cd}\end{array}$ & $\begin{array}{c}14.2 \\
\text { c }\end{array}$ & $\begin{array}{c}42.6 \\
h\end{array}$ & $\begin{array}{c}42.6 \\
h\end{array}$ & $\begin{array}{c}42.6 \\
h\end{array}$ & $\begin{array}{c}43.6 \\
d\end{array}$ & $\begin{array}{c}54.0 \\
\text { de }\end{array}$ & $\begin{array}{c}54.0 \\
\text { de }\end{array}$ & $\begin{array}{c}54.0 \\
\mathrm{de}\end{array}$ & $\begin{array}{c}54.0 \\
\text { de }\end{array}$ \\
\hline $0.2 \%$ & $\begin{array}{c}14.6 \\
\mathrm{~cd}\end{array}$ & $\begin{array}{c}18.1 \\
\mathrm{a} \\
\end{array}$ & $\begin{array}{c}17.3 \\
\mathrm{ab}\end{array}$ & $\begin{array}{c}16.7 \\
\text { ab }\end{array}$ & $\begin{array}{c}44.8 \\
\text { fgh }\end{array}$ & $\begin{array}{r}46.7 \\
\text { def }\end{array}$ & $\begin{array}{c}49.0 \\
\mathrm{bc}\end{array}$ & $\begin{array}{c}46.8 \\
\text { b }\end{array}$ & $\begin{array}{c}47.2 \\
\mathrm{f}\end{array}$ & $\begin{array}{c}55.0 \\
\mathrm{~d}\end{array}$ & $\begin{array}{c}54.0 \\
\mathrm{de}\end{array}$ & $\begin{array}{c}52.1 \\
\text { e }\end{array}$ \\
\hline $0.4 \%$ & $\begin{array}{c}17.7 \\
\mathrm{ab}\end{array}$ & $\begin{array}{c}17.4 \\
\mathrm{ab}\end{array}$ & $\begin{array}{c}15.9 \\
\text { bc }\end{array}$ & $\begin{array}{c}17.0 \\
\mathbf{a}\end{array}$ & $\begin{array}{l}44.8 \\
\text { fgh }\end{array}$ & $\begin{array}{l}46.5 \\
\text { defg }\end{array}$ & $\begin{array}{l}45.5 \\
0 \text { efg }\end{array}$ & $\begin{array}{c}45.6 \\
1 \\
\text { c }\end{array}$ & $\begin{array}{l}53.1 \\
7 \mathrm{de}\end{array}$ & $\begin{array}{l}58.6 \\
7 \mathrm{bc}\end{array}$ & $\begin{array}{l}54.3 \\
3 \mathrm{de}\end{array}$ & $\begin{array}{l}55.3 \\
9 \text { bc }\end{array}$ \\
\hline $0.6 \%$ & $\begin{array}{c}17.4 \\
\mathrm{ab}\end{array}$ & $\begin{array}{c}17.1 \\
\mathrm{~b}\end{array}$ & $\begin{array}{c}18.0 \\
\mathrm{a}\end{array}$ & $\begin{array}{c}17.5 \\
\mathrm{a}\end{array}$ & $\begin{array}{c}44.5 \\
\text { gh }\end{array}$ & $\begin{array}{c}52.0 \\
a\end{array}$ & $\begin{array}{c}51.8 \\
\mathrm{a}\end{array}$ & $\begin{array}{c}49.4 \\
6 \mathrm{a}\end{array}$ & $\begin{array}{c}53.6 \\
\text { de }\end{array}$ & $\begin{array}{c}60.6 \\
a b\end{array}$ & $\begin{array}{c}62.5 \\
a\end{array}$ & $\begin{array}{c}58.9 \\
a\end{array}$ \\
\hline $0.8 \%$ & $\begin{array}{c}15.3 \\
\mathrm{c}\end{array}$ & $\begin{array}{c}16.3 \\
\mathrm{~b}\end{array}$ & $\begin{array}{c}15.0 \\
\mathrm{c}\end{array}$ & $\begin{array}{c}15.5 \\
\text { bc }\end{array}$ & $\begin{array}{l}47.3 \\
\text { cde }\end{array}$ & $\begin{array}{c}50.0 \\
\mathrm{ab}\end{array}$ & $\begin{array}{c}40.8 \\
\mathrm{i}\end{array}$ & $\begin{array}{l}46.0 \\
4 \text { bc }\end{array}$ & $\begin{array}{c}58.1 \\
\mathrm{c}\end{array}$ & $\begin{array}{c}57.5 \\
\mathrm{c}\end{array}$ & $\begin{array}{c}52.2 \\
\mathrm{e}\end{array}$ & $\begin{array}{c}55.9 \\
\text { b }\end{array}$ \\
\hline $1 \%$ & $\begin{array}{c}16.6 \\
b\end{array}$ & $\begin{array}{c}18.3 \\
\mathrm{a}\end{array}$ & $\begin{array}{c}15.1 \\
\mathrm{c}\end{array}$ & $\begin{array}{c}16.7 \\
\text { ab }\end{array}$ & $\begin{array}{l}46.7 \\
\text { defg }\end{array}$ & $\begin{array}{c}40.0 \\
\mathrm{i}\end{array}$ & $\begin{array}{l}48.3 \\
\text { bcd }\end{array}$ & $\begin{array}{c}45.0 \\
\text { c }\end{array}$ & $\begin{array}{c}53.6 \\
\text { de }\end{array}$ & $\begin{array}{c}54.3 \\
\mathrm{de}\end{array}$ & $\begin{array}{c}55.1 \\
\mathrm{~d}\end{array}$ & $\begin{array}{c}54.3 \\
\text { cd }\end{array}$ \\
\hline Mean & $\begin{array}{c}16.0 \\
\text { a }\end{array}$ & $\begin{array}{c}16.9 \\
\text { a }\end{array}$ & $\begin{array}{c}15.9 \\
\text { a }\end{array}$ & & $\begin{array}{c}45.3 \\
\text { b }\end{array}$ & $\begin{array}{c}46.4 \\
\text { a }\end{array}$ & $\begin{array}{c}46.5 \\
\text { a }\end{array}$ & & $\begin{array}{c}53.3 \\
\text { c }\end{array}$ & $\begin{array}{c}56.6 \\
\text { a }\end{array}$ & $\begin{array}{c}55.3 \\
\text { b }\end{array}$ & \\
\hline
\end{tabular}

Different letters are significantly different at $p<0.05$. 
Table (3): Effect of different foliar spray concentrations (\% w: v) of three algal species on nodules number and $\mathrm{N}_{2 \text { - }}$ ase enzyme activity of faba bean root through growth stages of plant: A (45 days), B (75 days) and C (120 days). Data expressed as a mean of three replicates.

\begin{tabular}{|c|c|c|c|c|c|c|c|c|c|c|c|c|}
\hline \multirow{3}{*}{ 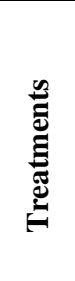 } & \multicolumn{12}{|c|}{ Nodules number } \\
\hline & \multicolumn{4}{|c|}{ Stage A } & \multicolumn{4}{|c|}{ Stage B } & \multicolumn{4}{|c|}{ Stage C } \\
\hline & 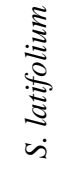 & 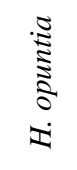 & 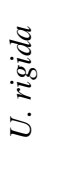 & 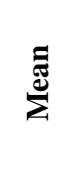 & 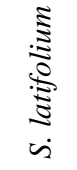 & 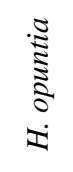 & 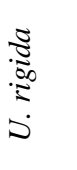 & $\sum^{\text {Јี }}$ & 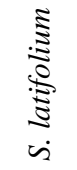 & 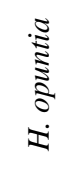 & 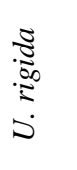 & $\sum_{\Sigma}^{\mathbb{E}}$ \\
\hline Cont. & $\begin{array}{c}56 \\
d\end{array}$ & $\begin{array}{c}56 \\
d\end{array}$ & $\begin{array}{c}56 \\
d\end{array}$ & $\begin{array}{c}56 \\
d\end{array}$ & $\begin{array}{c}71.56 \\
\mathrm{~g}\end{array}$ & $\begin{array}{c}71.56 \\
\mathrm{~g}\end{array}$ & $\begin{array}{c}71.56 \\
\mathrm{~g}\end{array}$ & $\begin{array}{c}71.56 \\
\text { e }\end{array}$ & $\begin{array}{c}45.0 \\
\mathrm{e}\end{array}$ & $\begin{array}{c}45.0 \\
\mathrm{e}\end{array}$ & $\begin{array}{c}45.0 \\
\mathrm{e}\end{array}$ & $\begin{array}{c}45.0 \\
\text { e }\end{array}$ \\
\hline $0.2 \%$ & $\begin{array}{l}64 \\
c d\end{array}$ & $\begin{array}{c}57 \\
d\end{array}$ & $\begin{array}{c}78 \\
\mathrm{c}\end{array}$ & $\begin{array}{c}66.33 \\
\text { c }\end{array}$ & $\begin{array}{c}78.0 \\
\text { fg }\end{array}$ & $\begin{array}{c}78.0 \\
\text { fg }\end{array}$ & $\begin{array}{c}97.0 \\
\text { de }\end{array}$ & $\begin{array}{c}84.33 \\
\text { d }\end{array}$ & $\begin{array}{c}80.0 \\
\text { b }\end{array}$ & $\begin{array}{c}70.0 \\
\mathrm{c}\end{array}$ & $\begin{array}{c}50.0 \\
\mathrm{e}\end{array}$ & $\begin{array}{c}66.67 \\
\text { c }\end{array}$ \\
\hline $0.4 \%$ & $\begin{array}{r}65 \\
\mathrm{c}\end{array}$ & $\begin{array}{c}64 \\
c\end{array}$ & $\begin{array}{c}78 \\
\mathrm{c}\end{array}$ & $\begin{array}{c}69 \\
\text { c }\end{array}$ & $\begin{array}{l}86.0 \\
\text { ef }\end{array}$ & $\begin{array}{c}101.6 \\
7 \mathrm{~cd}\end{array}$ & $\begin{array}{c}97.0 \\
\text { de }\end{array}$ & $\begin{array}{c}94.89 \\
\text { c }\end{array}$ & $\begin{array}{c}78.0 \\
\text { bc }\end{array}$ & $\begin{array}{c}50.0 \\
\mathrm{e}\end{array}$ & $\begin{array}{c}59.33 \\
\mathrm{~d}\end{array}$ & $\begin{array}{c}62.44 \\
\text { c }\end{array}$ \\
\hline $0.6 \%$ & $\begin{array}{c}75 \\
\text { c }\end{array}$ & $\begin{array}{r}81 \\
\mathrm{c}\end{array}$ & $\begin{array}{c}97 \\
b\end{array}$ & $\begin{array}{c}84.33 \\
\text { b }\end{array}$ & $\begin{array}{c}97.0 \\
\mathrm{de}\end{array}$ & $\begin{array}{c}105.6 \\
7 \mathrm{~cd}\end{array}$ & $\begin{array}{c}102.0 \\
\mathrm{~cd}\end{array}$ & $\begin{array}{c}101.5 \\
\text { cd }\end{array}$ & $\begin{array}{c}80.67 \\
\text { d }\end{array}$ & $\begin{array}{c}60.0 \\
\mathrm{~d}\end{array}$ & $\begin{array}{c}75.0 \\
\text { bc }\end{array}$ & $\begin{array}{c}71.89 \\
\text { b }\end{array}$ \\
\hline $0.8 \%$ & $\begin{array}{c}75 \\
c\end{array}$ & $\begin{array}{c}111 \\
a b\end{array}$ & $\begin{array}{c}115 \\
\mathrm{a}\end{array}$ & $\begin{array}{c}100.3 \\
\mathrm{a}\end{array}$ & $\begin{array}{c}93.00 \\
\text { de }\end{array}$ & $\begin{array}{c}122.0 \\
a b\end{array}$ & $\begin{array}{c}130.0 \\
\mathrm{a}\end{array}$ & $\begin{array}{c}115.0 \\
\text { a }\end{array}$ & $\begin{array}{c}100.0 \\
\mathrm{a}\end{array}$ & $\begin{array}{c}80.00 \\
b\end{array}$ & $\begin{array}{c}80.00 \\
\text { b }\end{array}$ & $\begin{array}{c}86.67 \\
a\end{array}$ \\
\hline $1 \%$ & $\begin{array}{c}76.67 \\
\mathrm{c} \\
\end{array}$ & $\begin{array}{c}110 \\
\mathrm{ab}\end{array}$ & $\begin{array}{c}120 \\
\mathrm{a}\end{array}$ & $\begin{array}{c}102.2 \\
\mathrm{a}\end{array}$ & $\begin{array}{c}78.33 \\
\text { fg } \\
\end{array}$ & $\begin{array}{c}113.0 \\
\text { bc }\end{array}$ & $\begin{array}{c}125.0 \\
\mathrm{ab}\end{array}$ & $\begin{array}{c}105.4 \\
\text { b }\end{array}$ & $\begin{array}{c}48.00 \\
\mathrm{e}\end{array}$ & $\begin{array}{c}80.00 \\
\text { b }\end{array}$ & $\begin{array}{c}70.00 \\
\mathrm{c}\end{array}$ & $\begin{array}{c}66.00 \\
c \\
\end{array}$ \\
\hline Mean & $\begin{array}{c}68.61 \\
\text { c }\end{array}$ & $\begin{array}{c}79.83 \\
\text { b }\end{array}$ & $\begin{array}{c}90.67 \\
\mathrm{a} \\
\end{array}$ & & $\begin{array}{c}84.06 \\
\text { b }\end{array}$ & $\begin{array}{c}98.72 \\
\mathrm{a} \\
\end{array}$ & $\begin{array}{c}103.8 \\
\mathrm{a} \\
\end{array}$ & & $\begin{array}{c}71.94 \\
\mathrm{a} \\
\end{array}$ & $\begin{array}{c}64.17 \\
\text { b }\end{array}$ & $\begin{array}{c}63.22 \\
\text { b }\end{array}$ & \\
\hline \multicolumn{13}{|c|}{ Nitroginase activity $\left(\mu \mathrm{mol} \mathrm{C}_{2} \mathrm{H}_{4} / \mathrm{h} / \mathrm{g}\right.$ dry nodules) } \\
\hline Cont. & $\begin{array}{c}218.7 \\
\mathrm{f}\end{array}$ & $\begin{array}{c}218.7 \\
\text { f }\end{array}$ & $\begin{array}{c}218.7 \\
\text { f }\end{array}$ & $\begin{array}{c}218.7 \\
\text { d }\end{array}$ & $\begin{array}{c}5111 \\
\mathrm{~h}\end{array}$ & $\begin{array}{c}5111 \\
\mathrm{~h}\end{array}$ & $\begin{array}{c}5111 \\
\mathrm{~h}\end{array}$ & $\begin{array}{c}5111 \\
\text { h }\end{array}$ & $\begin{array}{c}1074 \\
\mathrm{e}\end{array}$ & $\begin{array}{c}1074 \\
\mathrm{e}\end{array}$ & $\begin{array}{c}1074 \\
\mathrm{e}\end{array}$ & $\begin{array}{c}1074 \\
\text { d }\end{array}$ \\
\hline $0.2 \%$ & $\begin{array}{c}251.6 \\
\mathrm{e}\end{array}$ & $\begin{array}{c}416.2 \\
d\end{array}$ & $\begin{array}{c}543.8 \\
\mathrm{a}\end{array}$ & $\begin{array}{c}403.8 \\
\text { a }\end{array}$ & $\begin{array}{c}8581 . \\
3 \mathrm{fg}\end{array}$ & $\begin{array}{c}12675 \\
.3 \mathrm{de}\end{array}$ & $\begin{array}{c}982.1 \\
\mathrm{i}\end{array}$ & $\begin{array}{l}7412 . \\
91 \mathrm{~d}\end{array}$ & $\begin{array}{c}13648 \\
.8 \mathrm{~d}\end{array}$ & $\begin{array}{c}1210 . \\
6 \mathrm{e}\end{array}$ & $\begin{array}{c}1856 \\
\mathrm{e}\end{array}$ & $\begin{array}{c}5571 \\
\text { c }\end{array}$ \\
\hline $0.4 \%$ & $\begin{array}{c}507.2 \\
\text { b }\end{array}$ & $\begin{array}{c}466.1 \\
\mathrm{c}\end{array}$ & $\begin{array}{c}125.5 \\
\mathrm{~g}\end{array}$ & $\begin{array}{c}366.3 \\
\text { b }\end{array}$ & $\begin{array}{c}14840 \\
\text { d }\end{array}$ & $\begin{array}{c}31119 \\
b\end{array}$ & $\begin{array}{c}542.3 \\
\mathrm{i}\end{array}$ & $\begin{array}{c}15500 \\
\text { b }\end{array}$ & $\begin{array}{c}17060 \\
\mathrm{c}\end{array}$ & $\begin{array}{c}519.1 \\
\mathrm{e}\end{array}$ & $\begin{array}{c}15712 \\
\mathrm{a}\end{array}$ & $\begin{array}{c}58233 \\
a\end{array}$ \\
\hline $0.6 \%$ & $\begin{array}{c}220.0 \\
\mathrm{f}\end{array}$ & $\begin{array}{c}218.2 \\
\mathrm{f}\end{array}$ & $\begin{array}{c}56.66 \\
\mathrm{~h}\end{array}$ & $\begin{array}{c}164.9 \\
\mathrm{e}\end{array}$ & $\begin{array}{c}13848 \\
\mathrm{~d}\end{array}$ & $\begin{array}{c}31117 \\
b\end{array}$ & $\begin{array}{c}49017 \\
\text { a }\end{array}$ & $\begin{array}{c}31327 \\
\text { a }\end{array}$ & $\begin{array}{c}32627 \\
b\end{array}$ & $\begin{array}{c}1871 \\
\mathrm{e}\end{array}$ & $\begin{array}{c}2666 \\
\mathrm{e}\end{array}$ & $\begin{array}{c}12388 \\
\text { b }\end{array}$ \\
\hline $0.8 \%$ & $\begin{array}{c}219.4 \\
\mathrm{f}\end{array}$ & $\begin{array}{c}513.6 \\
\text { b }\end{array}$ & $\begin{array}{c}133.3 \\
\mathrm{~g}\end{array}$ & $\begin{array}{c}288.8 \\
\text { c }\end{array}$ & $\begin{array}{c}4374 \\
\mathrm{~h}\end{array}$ & $\begin{array}{c}4937 \\
\mathrm{~h}\end{array}$ & $\begin{array}{c}17793 \\
\mathrm{c}\end{array}$ & $\begin{array}{c}9034 \\
\text { c }\end{array}$ & $\begin{array}{c}34564 \\
.4 \mathrm{~b}\end{array}$ & $\begin{array}{c}2851 . \\
44 \mathrm{e}\end{array}$ & $\begin{array}{c}2589 \\
\mathrm{e}\end{array}$ & $\begin{array}{c}13334 \\
\text { b }\end{array}$ \\
\hline $1 \%$ & $\begin{array}{c}218.1 \\
\mathrm{f}\end{array}$ & $\begin{array}{c}424.0 \\
\mathrm{~d}\end{array}$ & $\begin{array}{c}234.0 \\
\text { ef }\end{array}$ & $\begin{array}{c}292.0 \\
\text { c }\end{array}$ & $\begin{array}{c}4870 \\
\mathrm{~h}\end{array}$ & $\begin{array}{c}7982 \\
\mathrm{~g}\end{array}$ & $\begin{array}{c}10789 \\
\text { ef }\end{array}$ & $\begin{array}{c}7880 \\
\text { cd }\end{array}$ & $\begin{array}{c}744.4 \\
\mathrm{e}\end{array}$ & $\begin{array}{c}1075 \\
\mathrm{e}\end{array}$ & $\begin{array}{c}1382 \\
\mathrm{e}\end{array}$ & $\begin{array}{c}1067 \\
\text { d }\end{array}$ \\
\hline Mean & $\begin{array}{c}272.5 \\
\text { b }\end{array}$ & $\begin{array}{c}376.1 \\
\mathrm{a} \\
\end{array}$ & $\begin{array}{c}218.6 \\
\text { c }\end{array}$ & & $\begin{array}{c}8604 \\
\text { c }\end{array}$ & $\begin{array}{c}15490 \\
\mathrm{a} \\
\end{array}$ & $\begin{array}{c}14039 \\
\text { b }\end{array}$ & & $\begin{array}{c}16619 \\
\text { b }\end{array}$ & $\begin{array}{c}1433 \\
\text { c }\end{array}$ & $\begin{array}{c}27781 \\
\text { a } \\
\end{array}$ & \\
\hline
\end{tabular}

Different letters are significantly different at $p<0.05$. 
Table (4): Effect of different foliar spray concentrations ( $\% \mathrm{w}: \mathrm{v}$ ) of three algal species on faba bean pigment contents ( $\mathrm{mg} / \mathrm{g}$ dry weight) at stage $A$ (45 days) and stage B (75 days). Data expressed as a mean of three replicates.

\begin{tabular}{|c|c|c|c|c|c|c|c|c|}
\hline \multirow[b]{2}{*}{ } & \multicolumn{4}{|c|}{ Stage A } & \multicolumn{4}{|c|}{ Stage B } \\
\hline & 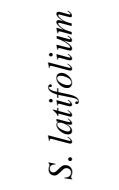 & 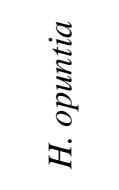 & 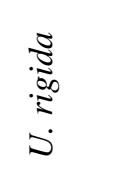 & $\sum_{\Sigma}^{\Xi}$ & 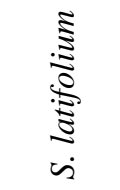 & 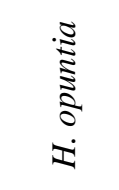 & $\begin{array}{l}\sqrt[3]{5} \\
-00 \\
0 \\
0 \\
0\end{array}$ & 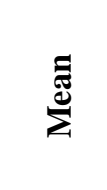 \\
\hline \multicolumn{9}{|c|}{ Chlorophyll a } \\
\hline Control & $6.90 \mathrm{de}$ & $6.90 \mathrm{de}$ & $6.90 \mathrm{de}$ & $6.90 \mathrm{bc}$ & $5.90 \mathrm{de}$ & $5.90 \mathrm{de}$ & $5.90 \mathrm{de}$ & $5.90 \mathrm{~d}$ \\
\hline $0.2 \%$ & $8.10 \mathrm{bc}$ & $7.58 \mathrm{~cd}$ & $7.99 \mathrm{bc}$ & $7.89 \mathrm{a}$ & $6.86 \mathrm{~b}$ & $6.63 \mathrm{~b}$ & $5.03 \mathrm{~g}$ & $6.17 \mathrm{bc}$ \\
\hline $0.4 \%$ & $6.40 \mathrm{e}$ & $7.92 \mathrm{bc}$ & $8.64 \mathrm{ab}$ & $7.65 \mathrm{a}$ & $6.76 \mathrm{~b}$ & $6.56 \mathrm{bc}$ & $5.34 \mathrm{fg}$ & $6.22 \mathrm{bc}$ \\
\hline $0.6 \%$ & $8.60 \mathrm{ab}$ & $8.98 \mathrm{a}$ & $6.40 \mathrm{e}$ & $7.99 \mathrm{a}$ & $6.55 \mathrm{bc}$ & $6.56 \mathrm{bc}$ & $8.56 \mathrm{a}$ & $7.22 \mathrm{a}$ \\
\hline $0.8 \%$ & $7.76 \mathrm{c}$ & $6.30 \mathrm{e}$ & $7.52 \mathrm{~cd}$ & $7.19 \mathrm{~b}$ & $5.59 \mathrm{ef}$ & $6.57 \mathrm{bc}$ & $6.95 \mathrm{~b}$ & $6.37 \mathrm{~b}$ \\
\hline $1 \%$ & $4.78 \mathrm{f}$ & $6.94 \mathrm{de}$ & $7.78 \mathrm{c}$ & $6.50 \mathrm{c}$ & $6.18 \mathrm{~cd}$ & $6.10 \mathrm{~d}$ & $5.88 \mathrm{de}$ & $6.05 \mathrm{~cd}$ \\
\hline Mean & $7.09 \mathrm{~b}$ & $7.44 \mathrm{a}$ & $7.54 \mathrm{a}$ & & $6.31 \mathrm{a}$ & $6.39 \mathrm{a}$ & $6.28 \mathrm{a}$ & \\
\hline \multicolumn{9}{|c|}{ Chlorophyll b } \\
\hline Control & $\begin{array}{l}2.60 \\
\text { efgh }\end{array}$ & $\begin{array}{l}2.60 \\
\text { efgh }\end{array}$ & $\begin{array}{l}2.60 \\
\text { efgh }\end{array}$ & $2.60 \mathrm{~cd}$ & $2.82 \mathrm{e}$ & $2.82 \mathrm{e}$ & $2.82 \mathrm{e}$ & $2.82 \mathrm{e}$ \\
\hline $0.2 \%$ & $\begin{array}{l}2.98 \\
\text { cde }\end{array}$ & $\begin{array}{l}3.06 \\
\text { bcd }\end{array}$ & $2.87 \mathrm{def}$ & $2.97 \mathrm{~b}$ & $3.48 \mathrm{abc}$ & $\begin{array}{l}3.42 \\
\text { abcd }\end{array}$ & $2.31 \mathrm{f}$ & $3.07 \mathrm{c}$ \\
\hline $0.4 \%$ & $2.24 \mathrm{~h}$ & $\begin{array}{l}2.53 \\
\text { efgh }\end{array}$ & $3.30 \mathrm{bc}$ & $2.69 \mathrm{~cd}$ & $\begin{array}{l}3.39 \\
\text { abcd }\end{array}$ & $3.19 \mathrm{~d}$ & $2.81 \mathrm{e}$ & 3.13 bc \\
\hline $0.6 \%$ & $3.88 \mathrm{a}$ & $3.38 \mathrm{~b}$ & $2.42 \mathrm{gh}$ & $3.23 \mathrm{a}$ & $3.25 \mathrm{~cd}$ & $3.19 \mathrm{~d}$ & $\begin{array}{l}3.31 \\
\text { bed }\end{array}$ & $3.25 \mathrm{ab}$ \\
\hline $0.8 \%$ & $\begin{array}{l}3.20 \\
\text { bcd }\end{array}$ & $2.38 \mathrm{~h}$ & $\begin{array}{l}2.82 \\
\text { defg }\end{array}$ & $2.80 \mathrm{bc}$ & $2.70 \mathrm{e}$ & $3.64 \mathrm{a}$ & $3.54 \mathrm{ab}$ & 3.29 a \\
\hline $1 \%$ & $2.20 \mathrm{~h}$ & $\begin{array}{l}2.48 \\
\text { fgh }\end{array}$ & $\begin{array}{l}2.81 \\
\text { defg }\end{array}$ & $2.50 \mathrm{~d}$ & $2.66 \mathrm{e}$ & $2.86 \mathrm{e}$ & $2.71 \mathrm{e}$ & $2.74 \mathrm{~d}$ \\
\hline Mean & $2.85 \mathrm{a}$ & $2.74 \mathrm{a}$ & $2.80 \mathrm{a}$ & & $3.05 \mathrm{~b}$ & $3.19 \mathrm{a}$ & $2.92 \mathrm{c}$ & \\
\hline \multicolumn{9}{|c|}{ Carotenoid } \\
\hline Control & $1.7 \mathrm{~h}$ & $1.7 \mathrm{~h}$ & $1.7 \mathrm{~h}$ & $1.7 \mathrm{c}$ & $1.61 \mathrm{~b}$ & $1.61 \mathrm{~b}$ & $1.61 \mathrm{~b}$ & $1.61 \mathrm{~b}$ \\
\hline $0.2 \%$ & $2.30 \mathrm{~cd}$ & $2.1 \mathrm{def}$ & $2.30 \mathrm{~cd}$ & $2.23 \mathrm{a}$ & $1.63 \mathrm{~b}$ & $1.56 \mathrm{~b}$ & $1.38 \mathrm{c}$ & $1.52 \mathrm{~cd}$ \\
\hline $0.4 \%$ & $1.66 \mathrm{~h}$ & $\begin{array}{l}2.18 \\
\text { cde }\end{array}$ & $2.63 \mathrm{a}$ & $2.16 \mathrm{a}$ & $1.58 \mathrm{~b}$ & $1.68 \mathrm{~b}$ & $1.22 \mathrm{~cd}$ & $1.49 \mathrm{~cd}$ \\
\hline $0.6 \%$ & $\begin{array}{l}2.22 \\
\text { cde }\end{array}$ & $2.58 \mathrm{ab}$ & $2.02 \mathrm{efg}$ & 2.27 a & $1.58 \mathrm{~b}$ & $1.68 \mathrm{~b}$ & $1.11 \mathrm{~d}$ & $1.46 \mathrm{~d}$ \\
\hline $0.8 \%$ & $1.92 \mathrm{fg}$ & $1.82 \mathrm{gh}$ & $\begin{array}{l}2.24 \\
\text { cde }\end{array}$ & $1.99 \mathrm{~b}$ & $1.63 \mathrm{~b}$ & $2.31 \mathrm{a}$ & $1.35 \mathrm{c}$ & $1.76 \mathrm{a}$ \\
\hline $1 \%$ & $1.38 \mathrm{i}$ & $2.08 \mathrm{def}$ & $2.40 \mathrm{bc}$ & $1.95 \mathrm{~b}$ & $1.58 \mathrm{~b}$ & $1.54 \mathrm{~b}$ & $1.55 \mathrm{c}$ & $1.56 \mathrm{bc}$ \\
\hline Mean & $1.86 \mathrm{c}$ & $2.08 \mathrm{~b}$ & $2.22 \mathrm{a}$ & & $1.60 \mathrm{~b}$ & $1.73 \mathrm{a}$ & $1.37 \mathrm{c}$ & \\
\hline
\end{tabular}

Different letters are significantly different at $p<0.05$. 
Table (5): Effect of different foliar spray concentrations (\% w: v) of the three algal species on endogenous nitrogen, phosphorus and potassium concentrations of faba bean through growth stages A (45 days), B (75 days) and C (120 days). Data expressed as a mean of three replicates.

\begin{tabular}{|c|c|c|c|c|c|c|c|c|c|c|c|c|}
\hline \multirow{3}{*}{ } & \multicolumn{12}{|c|}{ Nitrogen (\%) } \\
\hline & \multicolumn{4}{|c|}{ Stage A } & \multicolumn{4}{|c|}{ Stage B } & \multicolumn{4}{|c|}{ Stage C } \\
\hline & 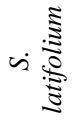 & 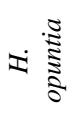 & 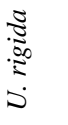 & $\sum_{\Sigma}^{\Xi}$ & 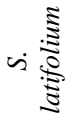 & 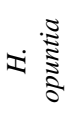 & 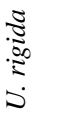 & 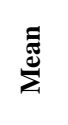 & 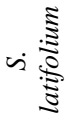 & 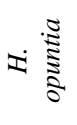 & 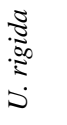 & 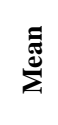 \\
\hline Cont. & $\begin{array}{l}2.66 \\
\text { efg }\end{array}$ & $\begin{array}{l}2.66 \\
\text { efg }\end{array}$ & $\begin{array}{c}2.66 \\
\text { efg }\end{array}$ & $\begin{array}{c}2.66 \\
\text { c }\end{array}$ & $\begin{array}{c}3.23 \\
\mathrm{a}\end{array}$ & $\begin{array}{c}3.23 \\
\mathrm{a}\end{array}$ & $\begin{array}{c}3.23 \\
\mathrm{a}\end{array}$ & $\begin{array}{c}3.23 \\
d\end{array}$ & $\begin{array}{c}1.90 \\
\text { ef }\end{array}$ & $\begin{array}{l}1.90 \\
\text { ef }\end{array}$ & $\begin{array}{c}1.90 \\
\text { ef }\end{array}$ & $\begin{array}{c}1.90 \\
\mathrm{e}\end{array}$ \\
\hline $0.2 \%$ & $\begin{array}{c}2.79 \\
\mathrm{de}\end{array}$ & $\begin{array}{c}2.72 \\
\text { def }\end{array}$ & $\begin{array}{c}2.79 \\
\text { de }\end{array}$ & $\begin{array}{c}2.77 \\
\text { c }\end{array}$ & $\begin{array}{c}3.42 \\
\mathrm{a}\end{array}$ & $\begin{array}{c}3.42 \\
\mathrm{a}\end{array}$ & $\begin{array}{c}3.80 \\
\mathrm{a}\end{array}$ & $\begin{array}{c}3.55 \\
\text { c }\end{array}$ & $\begin{array}{c}1.52 \\
\mathrm{~g}\end{array}$ & $\begin{array}{c}1.71 \\
\mathrm{f}\end{array}$ & $\begin{array}{c}1.90 \\
\text { ef }\end{array}$ & $\begin{array}{c}1.71 \\
\text { f }\end{array}$ \\
\hline $0.4 \%$ & $\begin{array}{l}2.98 \\
\text { bcd }\end{array}$ & $\begin{array}{l}3.17 \\
\text { abc }\end{array}$ & $\begin{array}{l}3.17 \\
\text { abc }\end{array}$ & $\begin{array}{c}3.10 \\
\text { b }\end{array}$ & $\begin{array}{c}3.61 \\
\mathrm{a}\end{array}$ & $\begin{array}{c}3.80 \\
\mathrm{a}\end{array}$ & $\begin{array}{c}4.18 \\
\mathrm{a}\end{array}$ & $\begin{array}{c}3.86 \\
\text { ab }\end{array}$ & $\begin{array}{c}2.28 \\
\mathrm{~cd}\end{array}$ & $\begin{array}{c}2.47 \\
\mathrm{c}\end{array}$ & $\begin{array}{c}2.47 \\
\mathrm{c}\end{array}$ & $\begin{array}{c}2.41 \\
b\end{array}$ \\
\hline $0.6 \%$ & $\begin{array}{c}3.23 \\
a b\end{array}$ & $\begin{array}{c}3.36 \\
\mathrm{a}\end{array}$ & $\begin{array}{c}3.36 \\
\mathrm{a}\end{array}$ & $\begin{array}{c}3.31 \\
\mathrm{a}\end{array}$ & $\begin{array}{c}3.80 \\
\mathrm{a}\end{array}$ & $\begin{array}{c}4.18 \\
\mathrm{a}\end{array}$ & $\begin{array}{c}4.04 \\
\mathrm{a}\end{array}$ & $\begin{array}{c}4.0 \\
a\end{array}$ & $\begin{array}{c}2.47 \\
\mathrm{c}\end{array}$ & $\begin{array}{c}2.66 \\
b\end{array}$ & $\begin{array}{c}2.85 \\
\mathrm{a}\end{array}$ & $\begin{array}{c}2.66 \\
a\end{array}$ \\
\hline $0.8 \%$ & $\begin{array}{l}2.91 \\
\text { cde }\end{array}$ & $\begin{array}{c}2.66 \\
\text { efg }\end{array}$ & $\begin{array}{c}2.47 \\
\text { fg }\end{array}$ & $\begin{array}{c}2.68 \\
\text { c }\end{array}$ & $\begin{array}{c}3.42 \\
\mathrm{a}\end{array}$ & $\begin{array}{c}3.61 \\
\mathrm{a}\end{array}$ & $\begin{array}{c}3.61 \\
\mathrm{a}\end{array}$ & $\begin{array}{c}3.55 \\
\text { c }\end{array}$ & $\begin{array}{c}2.09 \\
\mathrm{de}\end{array}$ & $\begin{array}{c}2.28 \\
\mathrm{~cd}\end{array}$ & $\begin{array}{c}2.09 \\
\mathrm{de}\end{array}$ & $\begin{array}{c}2.15 \\
\text { c }\end{array}$ \\
\hline $1 \%$ & $\begin{array}{c}2.41 \\
\mathrm{~g}\end{array}$ & $\begin{array}{c}2.79 \\
\text { de }\end{array}$ & $\begin{array}{l}2.91 \\
\text { cde }\end{array}$ & $\begin{array}{c}2.70 \\
\text { c }\end{array}$ & $\begin{array}{c}3.61 \\
\mathrm{a}\end{array}$ & $\begin{array}{c}3.42 \\
\mathrm{a}\end{array}$ & $\begin{array}{c}3.94 \\
3 \mathrm{a}\end{array}$ & $\begin{array}{l}3.66 \\
\text { bc }\end{array}$ & $\begin{array}{c}1.90 \\
\text { ef }\end{array}$ & $\begin{array}{c}1.90 \\
\text { ef }\end{array}$ & $\begin{array}{c}2.28 \\
\mathrm{~cd}\end{array}$ & $\begin{array}{c}2.03 \\
\text { d }\end{array}$ \\
\hline Mean & $\begin{array}{c}2.83 \\
\text { a }\end{array}$ & $\begin{array}{c}2.89 \\
\text { a }\end{array}$ & $\begin{array}{c}2.89 \\
\text { a }\end{array}$ & & $\begin{array}{c}3.52 \\
\text { b }\end{array}$ & $\begin{array}{c}3.61 \\
\text { b }\end{array}$ & $\begin{array}{c}3.80 \\
\text { a }\end{array}$ & & $\begin{array}{c}2.03 \\
\text { c }\end{array}$ & $\begin{array}{c}2.15 \\
\text { b }\end{array}$ & $\begin{array}{c}2.25 \\
\mathrm{a}\end{array}$ & \\
\hline \multicolumn{13}{|c|}{ Phosphorus (\%) } \\
\hline Cont. & $\begin{array}{c}0.17 \\
\mathrm{c} \\
\end{array}$ & $\begin{array}{c}0.17 \\
\mathrm{c} \\
\end{array}$ & $\begin{array}{c}0.17 \\
\mathrm{c} \\
\end{array}$ & $\begin{array}{c}0.17 \\
\text { d }\end{array}$ & $\begin{array}{c}0.76 \\
\mathrm{i} \\
\end{array}$ & $\begin{array}{c}0.76 \\
\mathrm{i} \\
\end{array}$ & $\begin{array}{c}0.76 \\
\mathrm{i} \\
\end{array}$ & $\begin{array}{c}0.76 \\
\text { d }\end{array}$ & $\begin{array}{c}0.11 \\
\text { de }\end{array}$ & $\begin{array}{c}0.11 \\
\text { de }\end{array}$ & $\begin{array}{c}0.11 \\
\mathrm{de}\end{array}$ & $\begin{array}{c}0.11 \\
\text { c }\end{array}$ \\
\hline $0.2 \%$ & $\begin{array}{c}0.25 \\
\mathrm{a}\end{array}$ & $\begin{array}{c}0.17 \\
1 \\
\mathrm{c} \\
\end{array}$ & $\begin{array}{c}0.17 \\
\mathrm{c}\end{array}$ & $\begin{array}{c}1.96 \\
\mathrm{c}\end{array}$ & $\begin{array}{c}0.98 \\
\text { gh }\end{array}$ & $\begin{array}{c}1.14 \\
\text { def }\end{array}$ & $\begin{array}{c}0.87 \\
\text { hi }\end{array}$ & $\begin{array}{c}0.99 \\
c\end{array}$ & $\begin{array}{c}0.11 \\
\mathrm{de}\end{array}$ & $\begin{array}{c}0.09 \\
\mathrm{e}\end{array}$ & $\begin{array}{c}0.11 \\
\text { de }\end{array}$ & $\begin{array}{c}0.10 \\
\text { c }\end{array}$ \\
\hline $0.4 \%$ & $\begin{array}{c}0.26 \\
\mathrm{a} \\
\end{array}$ & $\begin{array}{c}0.24 \\
\mathrm{a} \\
\end{array}$ & $\begin{array}{c}0.18 \\
\mathrm{c} \\
\end{array}$ & $\begin{array}{c}0.23 \\
\text { b }\end{array}$ & $\begin{array}{l}1.31 \\
\mathrm{abc}\end{array}$ & $\begin{array}{c}1.33 \\
\mathrm{ab}\end{array}$ & $\begin{array}{l}1.22 \\
\text { bcde }\end{array}$ & $\begin{array}{c}1.29 \\
\mathrm{a} \\
\end{array}$ & $\begin{array}{l}0.14 \\
\text { cde }\end{array}$ & $\begin{array}{c}0.17 \\
\text { bc }\end{array}$ & $\begin{array}{c}0.16 \\
b c\end{array}$ & $\begin{array}{c}0.16 \\
\text { b }\end{array}$ \\
\hline $0.6 \%$ & $\begin{array}{c}0.25 \\
\mathrm{a}\end{array}$ & $\begin{array}{c}0.24 \\
\mathrm{a}\end{array}$ & $\begin{array}{c}0.25 \\
\mathrm{a}\end{array}$ & $\begin{array}{c}0.25 \\
\mathrm{a}\end{array}$ & $\begin{array}{c}1.33 \\
a b\end{array}$ & $\begin{array}{c}1.41 \\
\mathrm{a}\end{array}$ & $\begin{array}{l}1.28 \\
\text { abcd }\end{array}$ & $\begin{array}{c}1.34 \\
\mathrm{a}\end{array}$ & $\begin{array}{c}0.16 \\
b c\end{array}$ & $\begin{array}{c}0.19 \\
b\end{array}$ & $\begin{array}{c}0.26 \\
\mathrm{a}\end{array}$ & $\begin{array}{c}0.20 \\
a\end{array}$ \\
\hline $0.8 \%$ & $\begin{array}{c}0.24 \\
\mathrm{a}\end{array}$ & $\begin{array}{c}0.21 \\
b\end{array}$ & $\begin{array}{c}0.16 \\
\mathrm{c}\end{array}$ & $\begin{array}{c}0.20 \\
\text { c }\end{array}$ & $\begin{array}{c}1.06 \\
\mathrm{fg}\end{array}$ & $\begin{array}{l}1.17 \\
\text { cdef }\end{array}$ & $\begin{array}{l}1.25 \\
\text { bcde }\end{array}$ & $\begin{array}{c}1.16 \\
b\end{array}$ & $\begin{array}{c}0.11 \\
\text { de }\end{array}$ & $\begin{array}{c}0.11 \\
\mathrm{de}\end{array}$ & $\begin{array}{c}0.11 \\
\text { de }\end{array}$ & $\begin{array}{c}0.11 \\
\text { c }\end{array}$ \\
\hline $1 \%$ & $\begin{array}{c}0.19 \\
\text { bc }\end{array}$ & $\begin{array}{c}0.16 \\
\mathrm{c}\end{array}$ & $\begin{array}{c}0.19 \\
\mathrm{c}\end{array}$ & $\begin{array}{c}\mathbf{0 . 1 8} \\
\text { d }\end{array}$ & $\begin{array}{l}1.11 \\
\text { efg }\end{array}$ & $\begin{array}{l}1.25 \\
\text { bcde }\end{array}$ & $\begin{array}{l}1.11 \\
\text { efg }\end{array}$ & $\begin{array}{c}1.16 \\
b\end{array}$ & $\begin{array}{l}0.14 \\
\text { cde }\end{array}$ & $\begin{array}{c}0.16 \\
b c\end{array}$ & $\begin{array}{l}0.15 \\
\text { bcd }\end{array}$ & $\begin{array}{c}0.15 \\
\text { b }\end{array}$ \\
\hline Mean & $\begin{array}{c}0.23 \\
\mathrm{a} \\
\end{array}$ & $\begin{array}{c}0.2 \\
\text { b }\end{array}$ & $\begin{array}{c}0.18 \\
\text { c } \\
\end{array}$ & & $\begin{array}{c}1.09 \\
\text { b }\end{array}$ & $\begin{array}{c}1.18 \\
a \\
\end{array}$ & $\begin{array}{c}1.08 \\
\text { b }\end{array}$ & & $\begin{array}{c}\mathbf{0 . 1 3} \\
\mathbf{a} \\
\end{array}$ & $\begin{array}{c}0.14 \\
\text { a } \\
\end{array}$ & $\begin{array}{c}0.15 \\
\mathrm{a} \\
\end{array}$ & \\
\hline & & & & & Pot & sium ( & & & & & & \\
\hline Cont. & $\begin{array}{c}1.47 \\
\mathrm{a} \\
\end{array}$ & $\begin{array}{c}1.47 \\
\mathrm{a} \\
\end{array}$ & $\begin{array}{c}1.47 \\
\mathrm{a} \\
\end{array}$ & $\begin{array}{c}1.47 \\
\text { b }\end{array}$ & $\begin{array}{c}1.42 \\
\mathrm{de}\end{array}$ & $\begin{array}{c}1.42 \\
\mathrm{de}\end{array}$ & $\begin{array}{c}1.42 \\
\mathrm{de}\end{array}$ & $\begin{array}{c}1.42 \\
\text { de }\end{array}$ & $\begin{array}{c}1.08 \\
\text { ef }\end{array}$ & $\begin{array}{c}1.08 \\
\text { ef }\end{array}$ & $\begin{array}{c}1.08 \\
\text { ef }\end{array}$ & $\begin{array}{c}1.08 \\
\text { b }\end{array}$ \\
\hline $0.2 \%$ & $\begin{array}{c}1.47 \\
\mathrm{a} \\
\end{array}$ & $\begin{array}{c}1.40 \\
\mathrm{a} \\
\end{array}$ & $\begin{array}{c}1.44 \\
\mathrm{a} \\
\end{array}$ & $\begin{array}{c}1.44 \\
b\end{array}$ & $\begin{array}{c}1.44 \\
\mathrm{de}\end{array}$ & $\begin{array}{c}1.37 \\
\mathrm{e}\end{array}$ & $\begin{array}{c}1.40 \\
\mathrm{de}\end{array}$ & $\begin{array}{c}1.40 \\
\text { c }\end{array}$ & $\begin{array}{c}1.22 \\
\mathrm{~cd}\end{array}$ & $\begin{array}{c}0.93 \\
\mathrm{~g}\end{array}$ & $\begin{array}{c}1.13 \\
\mathrm{de}\end{array}$ & $\begin{array}{c}1.09 \\
\text { b }\end{array}$ \\
\hline $0.4 \%$ & $\begin{array}{c}1.76 \\
\mathrm{a}\end{array}$ & $\begin{array}{c}1.75 \\
\mathrm{a}\end{array}$ & $\begin{array}{c}1.57 \\
\mathrm{a}\end{array}$ & $\begin{array}{c}1.69 \\
\text { a }\end{array}$ & $\begin{array}{c}1.65 \\
b c\end{array}$ & $\begin{array}{c}1.69 \\
\mathrm{ab}\end{array}$ & $\begin{array}{c}1.51 \\
\mathrm{de}\end{array}$ & $\begin{array}{c}1.62 \\
b\end{array}$ & $\begin{array}{c}1.44 \\
\mathrm{a}\end{array}$ & $\begin{array}{c}1.40 \\
\mathrm{ab}\end{array}$ & $\begin{array}{c}1.35 \\
a b\end{array}$ & $\begin{array}{c}1.39 \\
\text { a }\end{array}$ \\
\hline $0.6 \%$ & $\begin{array}{c}1.82 \\
\mathrm{a}\end{array}$ & $\begin{array}{c}1.57 \\
\mathrm{a}\end{array}$ & $\begin{array}{c}1.68 \\
\mathrm{a}\end{array}$ & $\begin{array}{c}1.69 \\
\text { a }\end{array}$ & $\begin{array}{c}1.76 \\
a b\end{array}$ & $\begin{array}{c}1.51 \\
\mathrm{de}\end{array}$ & $\begin{array}{c}1.79 \\
\mathrm{a}\end{array}$ & $\begin{array}{c}1.69 \\
a\end{array}$ & $\begin{array}{c}1.37 \\
\mathrm{ab}\end{array}$ & $\begin{array}{c}1.37 \\
\mathrm{ab}\end{array}$ & $\begin{array}{c}1.40 \\
a b\end{array}$ & $\begin{array}{c}1.38 \\
\mathrm{a}\end{array}$ \\
\hline $0.8 \%$ & $\begin{array}{c}1.62 \\
\mathrm{a}\end{array}$ & $\begin{array}{c}1.47 \\
\mathrm{a}\end{array}$ & $\begin{array}{c}1.53 \\
\mathrm{a}\end{array}$ & $\begin{array}{c}1.54 \\
b\end{array}$ & $\begin{array}{c}1.54 \\
\mathrm{~cd}\end{array}$ & $\begin{array}{c}1.44 \\
\mathrm{de}\end{array}$ & $\begin{array}{c}1.49 \\
\mathrm{de}\end{array}$ & $\begin{array}{c}1.49 \\
b\end{array}$ & $\begin{array}{c}1.31 \\
b c\end{array}$ & $\begin{array}{c}1.01 \\
\text { fg }\end{array}$ & $\begin{array}{l}1.11 \\
\text { def }\end{array}$ & $\begin{array}{c}1.14 \\
\text { b }\end{array}$ \\
\hline $1 \%$ & $\begin{array}{c}1.42 \\
\mathrm{a} \\
\end{array}$ & $\begin{array}{c}1.46 \\
\mathrm{a} \\
\end{array}$ & $\begin{array}{c}1.47 \\
\mathrm{a} \\
\end{array}$ & $\begin{array}{c}1.45 \\
\text { b } \\
\end{array}$ & $\begin{array}{c}1.40 \\
\text { de }\end{array}$ & $\begin{array}{c}1.49 \\
\text { de }\end{array}$ & $\begin{array}{c}1.44 \\
\mathrm{de}\end{array}$ & $\begin{array}{c}1.44 \\
\text { bc }\end{array}$ & $\begin{array}{c}1.24 \\
\mathrm{c}\end{array}$ & $\begin{array}{c}1.04 \\
\text { ef }\end{array}$ & $\begin{array}{c}1.08 \\
\text { ef }\end{array}$ & $\begin{array}{c}1.12 \\
\text { b }\end{array}$ \\
\hline Mean & $\begin{array}{c}1.59 \\
\text { a }\end{array}$ & $\begin{array}{c}1.52 \\
a\end{array}$ & $\begin{array}{c}1.53 \\
\mathrm{a}\end{array}$ & & $\begin{array}{c}1.53 \\
\text { a }\end{array}$ & $\begin{array}{c}1.49 \\
\mathrm{a}\end{array}$ & $\begin{array}{c}1.51 \\
\mathrm{a}\end{array}$ & & $\begin{array}{c}1.28 \\
\mathrm{a}\end{array}$ & $\begin{array}{c}1.14 \\
c\end{array}$ & $\begin{array}{c}1.19 \\
\text { b }\end{array}$ & \\
\hline
\end{tabular}

Different letters are significantly different at $p<0.05$. 
Table (6): Effect of different foliar spray concentrations (\% w: v) of three algal species on tillers number and pods number of faba bean; at stage $C$ (120 days). Data expressed as a mean of three replicates.

\begin{tabular}{|c|c|c|c|c|c|c|c|c|}
\hline \multirow[b]{2}{*}{ 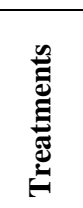 } & \multicolumn{4}{|c|}{ Tillers number } & \multicolumn{4}{|c|}{ Pods number } \\
\hline & 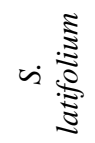 & 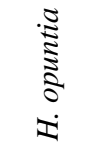 & 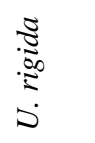 & 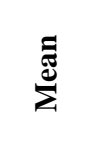 & 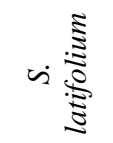 & 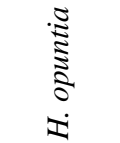 & 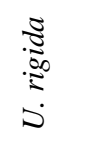 & $\stackrel{\Xi}{\stackrel{\Xi}{\Xi}}$ \\
\hline Contl & $1.33 \mathrm{a}$ & $1.33 \mathrm{a}$ & $1.33 \mathrm{a}$ & $1.33 \mathrm{a}$ & $4.50 \mathrm{gh}$ & $4.50 \mathrm{gh}$ & $4.50 \mathrm{gh}$ & $4.50 \mathrm{e}$ \\
\hline $0.2 \%$ & $1.17 \mathrm{a}$ & $1.33 \mathrm{a}$ & $1.33 \mathrm{a}$ & $1.28 \mathrm{a}$ & $3.67 \mathrm{i}$ & $5.50 \mathrm{e}$ & $4.83 \mathrm{f}$ & $4.67 \mathrm{e}$ \\
\hline $0.4 \%$ & $1.17 \mathrm{a}$ & $1.83 \mathrm{a}$ & $1.33 \mathrm{a}$ & $1.44 \mathrm{a}$ & $5.83 \mathrm{~d}$ & $7.00 \mathrm{~b}$ & $4.67 \mathrm{fg}$ & $5.83 \mathrm{~b}$ \\
\hline $0.6 \%$ & $1.00 \mathrm{a}$ & $1.33 \mathrm{a}$ & $1.00 \mathrm{a}$ & $1.11 \mathrm{a}$ & $5.33 \mathrm{e}$ & $7.50 \mathrm{a}$ & $6.33 \mathrm{c}$ & $6.39 \mathrm{a}$ \\
\hline $0.8 \%$ & $1.33 \mathrm{a}$ & $1.33 \mathrm{a}$ & $1.66 \mathrm{a}$ & $1.44 \mathrm{a}$ & $5.33 \mathrm{e}$ & $5.98 \mathrm{~d}$ & $4.33 \mathrm{~h}$ & $5.21 \mathrm{~d}$ \\
\hline $1 \%$ & $1.17 \mathrm{a}$ & $1.33 \mathrm{a}$ & $1.17 \mathrm{a}$ & $1.22 \mathrm{a}$ & $5.5 \mathrm{e}$ & $5.83 \mathrm{~d}$ & $5.33 \mathrm{e}$ & $5.55 \mathrm{c}$ \\
\hline Mean & $1.19 \mathrm{a}$ & $1.41 \mathrm{a}$ & $1.30 \mathrm{a}$ & & $5.03 \mathrm{~b}$ & $6.05 \mathrm{a}$ & $5.00 \mathrm{~b}$ & \\
\hline
\end{tabular}

Different letters are significantly different at $p<0.05$.
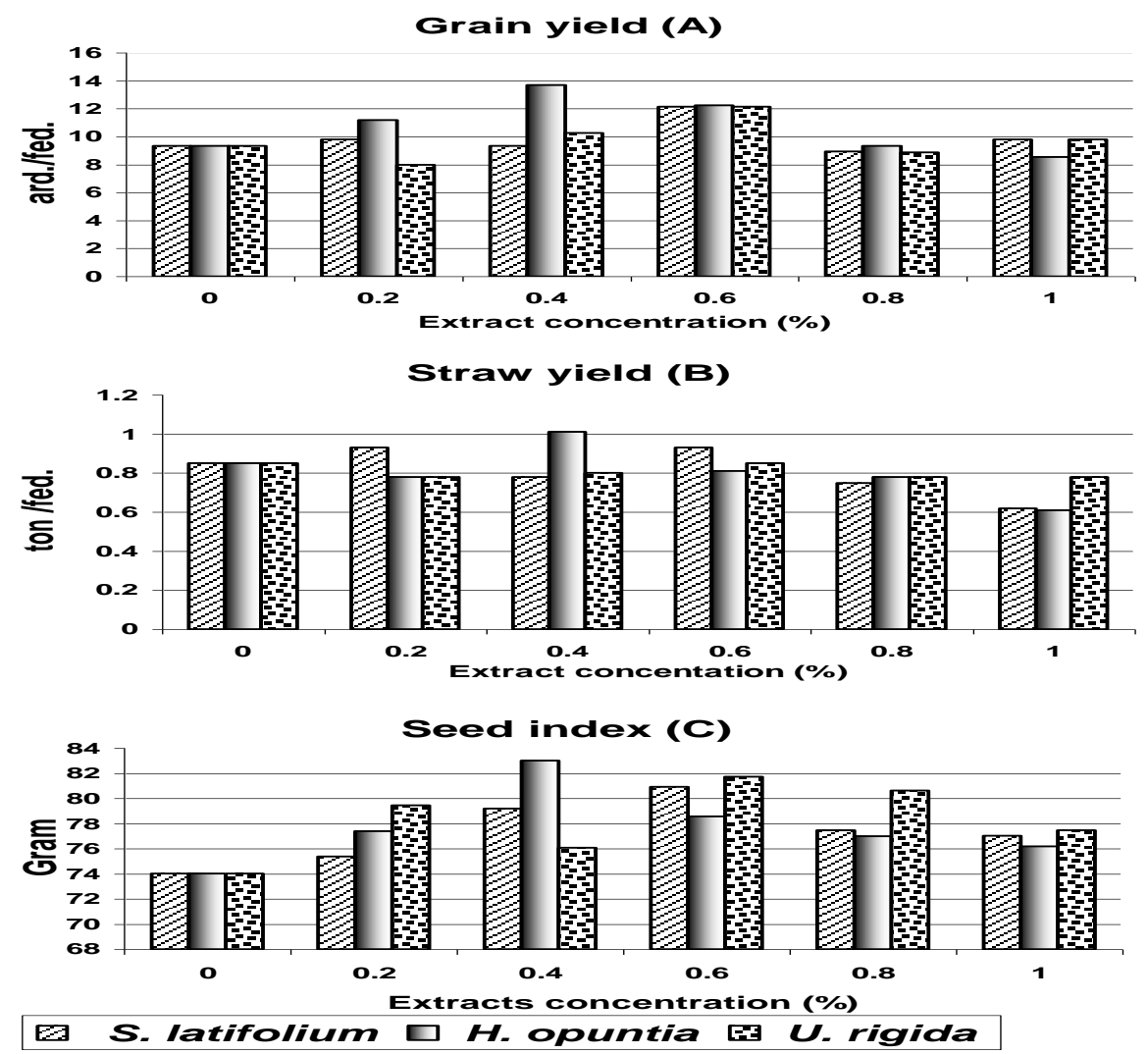

Figure (1): Effect of different foliar spray concentrations (\% w: v) of the algal species extract on yield parameters of faba bean after 120 days from sowing $A$ Grain yield, B- Straw yield and C-Seed index after 120 days. 
increased by foliar spray at concentration $0.6 \%$ for all the three studied algal species (Figure 2).

Also, the $0.6 \%$ concentration of foliar spray of the three studied algae species increased significantly the amount of NPK for faba bean seed (Table 7).
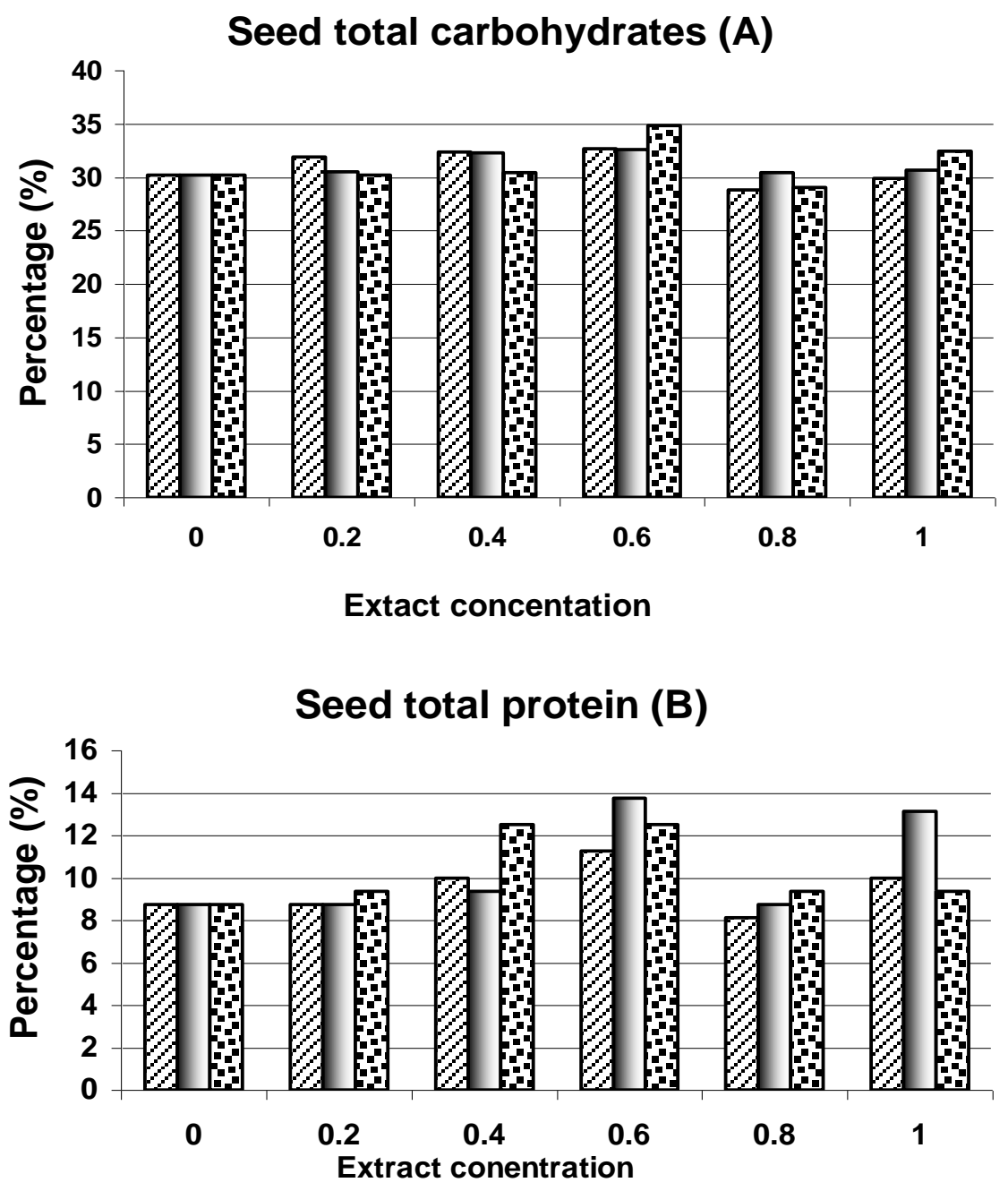

\section{$\square$ S. latifolium $\square$ H. opuntia $\mathbf{U}$. rigida}

Figure (2): Effect of different foliar spray concentrations (\% w: v) of the algal species extract on the frequency of total carbohydrates (A) and total protein (B) of faba bean seeds. 
Table (7): Effect of different foliar spray concentrations (\% w: v) of three algal species extracts on the frequency of total nitrogen, total phosphorus and total potassium of faba bean seeds. Data expressed as a mean of three replicates.

\begin{tabular}{|c|c|c|c|c|c|c|c|c|c|c|c|c|}
\hline \multirow[b]{2}{*}{ 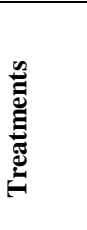 } & \multicolumn{4}{|c|}{ Total Nitrogen } & \multicolumn{4}{|c|}{ Total Phosphorus } & \multicolumn{4}{|c|}{ Total Potassium } \\
\hline & 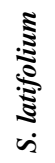 & 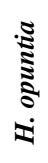 & 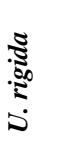 & $\stackrel{\text { త్ర }}{\sum^{\sharp}}$ & 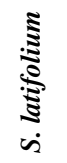 & 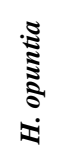 & 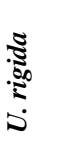 & 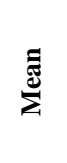 & 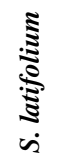 & 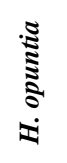 & 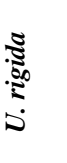 & 胥 \\
\hline $\begin{array}{c}\text { Contr } \\
\text { ol }\end{array}$ & $\begin{array}{c}1.4 \\
\text { ef }\end{array}$ & $\begin{array}{c}1.4 \\
\text { ef }\end{array}$ & $\begin{array}{l}1.4 \\
\text { ef }\end{array}$ & $\begin{array}{c}1.4 \\
\text { c }\end{array}$ & $\begin{array}{c}0.22 \\
\text { efg }\end{array}$ & $\begin{array}{c}0.22 \\
\text { efg }\end{array}$ & $\begin{array}{c}\begin{array}{c}0.22 \\
\text { efg }\end{array} \\
\end{array}$ & $\begin{array}{c}0.22 \\
\text { c }\end{array}$ & $\begin{array}{c}0.81 \\
a\end{array}$ & $\begin{array}{c}0.81 \\
a\end{array}$ & $\begin{array}{c}0.81 \\
a\end{array}$ & $\begin{array}{c}0.81 \\
\text { c }\end{array}$ \\
\hline $0.2 \%$ & $\begin{array}{c}1.4 \\
\text { ef }\end{array}$ & $\begin{array}{c}1.4 \\
\text { ef }\end{array}$ & $\begin{array}{l}1.5 \\
\text { de }\end{array}$ & $\begin{array}{c}1.4 \\
\text { c }\end{array}$ & $\begin{array}{c}0.22 \\
\text { efg }\end{array}$ & $\begin{array}{c}0.17 \\
\text { i }\end{array}$ & $\begin{array}{c}0.19 \\
\text { h }\end{array}$ & $\begin{array}{c}0.2 \\
\text { d }\end{array}$ & $\begin{array}{c}1.03 \\
a\end{array}$ & $\begin{array}{c}0.97 \\
\text { a }\end{array}$ & $\begin{array}{c}0.95 \\
\text { a }\end{array}$ & $\begin{array}{c}0.99 \\
\text { ab }\end{array}$ \\
\hline $0.4 \%$ & $\begin{array}{c}1.6 \\
\text { d }\end{array}$ & $\begin{array}{l}1.5 \\
\text { de }\end{array}$ & $\begin{array}{c}2.0 \\
\text { b }\end{array}$ & $\begin{array}{c}1.7 \\
\text { b }\end{array}$ & $\begin{array}{l}\text { O.21 } \\
\text { fgh }\end{array}$ & $\begin{array}{l}0.24 \\
\text { bed }\end{array}$ & $\begin{array}{c}0.24 \\
\text { cd }\end{array}$ & $\begin{array}{c}0.23 \\
\text { b }\end{array}$ & $\begin{array}{c}1.08 \\
\mathrm{a}\end{array}$ & $\begin{array}{c}1.03 \\
\mathrm{a}\end{array}$ & $\begin{array}{c}0.99 \\
\text { a } \\
\end{array}$ & $\begin{array}{c}1.03 \\
\mathrm{a}\end{array}$ \\
\hline $0.6 \%$ & $\begin{array}{c}1.8 \\
\text { c }\end{array}$ & $\begin{array}{c}2.2 \\
\mathrm{a}\end{array}$ & $\begin{array}{c}2.0 \\
\text { b }\end{array}$ & $\begin{array}{c}2.0 \\
\text { a }\end{array}$ & $\begin{array}{c}0.26 \\
\text { ab }\end{array}$ & $\begin{array}{c}0.23 \\
\text { de }\end{array}$ & $\begin{array}{l}0.26 \\
\text { abc }\end{array}$ & $\begin{array}{c}0.25 \\
\mathrm{a} \\
\end{array}$ & $\begin{array}{c}1.08 \\
\mathrm{a} \\
\end{array}$ & $\begin{array}{c}0.97 \\
\text { a }\end{array}$ & $\begin{array}{c}1.04 \\
\mathrm{a}\end{array}$ & $\begin{array}{c}1.03 \\
\mathrm{a} \\
\end{array}$ \\
\hline $0.8 \%$ & $\begin{array}{c}1.3 \\
\text { f }\end{array}$ & $\begin{array}{c}1.4 \\
\text { ef }\end{array}$ & $\begin{array}{l}1.5 \\
\text { de }\end{array}$ & $\begin{array}{c}1.4 \\
\mathrm{c}\end{array}$ & $\begin{array}{c}0.19 \\
\text { h }\end{array}$ & $\begin{array}{c}0.17 \\
\text { i }\end{array}$ & $\begin{array}{c}0.20 \\
\text { gh }\end{array}$ & $\begin{array}{c}0.19 \\
d\end{array}$ & $\begin{array}{c}0.95 \\
\text { a }\end{array}$ & $\begin{array}{c}0.97 \\
\text { a }\end{array}$ & $\begin{array}{c}0.97 \\
\text { a }\end{array}$ & $\begin{array}{c}0.97 \\
\text { b }\end{array}$ \\
\hline $1 \%$ & $\begin{array}{c}16 \\
d\end{array}$ & $\begin{array}{l}2.1 \\
\text { ab }\end{array}$ & $\begin{array}{l}1.5 \\
\text { de }\end{array}$ & $\begin{array}{c}1.73 \\
\text { b }\end{array}$ & $\begin{array}{c}0.22 \\
\text { ef }\end{array}$ & $\begin{array}{c}0.27 \\
\mathrm{a}\end{array}$ & $\begin{array}{c}0.20 \\
\text { gh }\end{array}$ & $\begin{array}{c}0.23 \\
\text { b }\end{array}$ & $\begin{array}{c}0.97 \\
\text { a }\end{array}$ & $\begin{array}{c}0.99 \\
\text { a }\end{array}$ & $\begin{array}{c}0.92 \\
\text { a }\end{array}$ & $\begin{array}{c}0.96 \\
b\end{array}$ \\
\hline Mean & $\begin{array}{l}1.5 \\
2 \mathrm{~b}\end{array}$ & $\begin{array}{l}1.6 \\
7 \mathrm{a}\end{array}$ & $\begin{array}{c}1.65 \\
a\end{array}$ & & $\begin{array}{c}0.22 \\
\mathrm{a}\end{array}$ & $\begin{array}{c}0.22 \\
\mathrm{a}\end{array}$ & $\begin{array}{c}0.22 \\
\mathrm{a}\end{array}$ & & $\begin{array}{c}0.99 \\
\text { a }\end{array}$ & $\begin{array}{c}0.96 \\
\text { a }\end{array}$ & $\begin{array}{c}0.96 \\
\text { b }\end{array}$ & \\
\hline
\end{tabular}

Different letters are significantly different at $p<0.05$.

\section{Discussion}

The micronutrients status of the soil is limited. Low rates of nutrients will not be sufficient to close the gap between crop need and supply. The continuous use of low rates of fertilizer nutrients can deplete the soil nutrient reserves, so the uses of seaweed provide the plants with mineral elements and stimulating the action of soil bacteria. The present investigation demonstrated that faba bean root criteria increased with foliar spray with seaweed extract, whereas shoot parameters were enhanced with foliar spray. Pigment content of faba bean was also increased on foliar spray, these results were in favor with the findings of Blunden et al. (1996) and Crouch and van Staden (1992) also found that the seaweed concentrate stimulated root growth at the expense of shoot growth and increase of the overall photosynthetic accumulation efficiency of the plant. A better root system might be attributed to endogenous auxin and related compounds in the extracts. The seaweed concentrate has also been shown to enhance nutrient uptake by roots. Foliar spray seaweed extracts may represent an alternative to supply the plant with micronutrients. C. prolifera had the high amount of $\mathrm{Cu}, \mathrm{Zn}, \mathrm{Cd}$ and $\mathrm{Mn}$.

In the present study it was clear that the algal extract increased the percentages of faba bean NPK concentration, especially at concentration $0.6 \%$. The present data showed that the yield of faba bean was increased by seaweed application, which agreed with the studies made by Featonby and van Staden 
(1984), Temple and Bomke (1989) and Beckett et al. (1994) on bean plants. They showed that seaweed extract did not act simply as a fertilizer, but as some kind of bio-stimulant. They suggested that seaweed extract could have increase yield in at least two ways. The first, it might have increase the source capacity of the leaves, thus increasing the supply of assimilates available for bean filling. This might by increasing leaf area and photosynthetic rates. Second, the seaweed extract might increase bean weight by enhancing the sink potential of the fruit for assimilates, thus increasing cotyledon cell number and final seed mass.

Addition of the algal extracts caused increase of chlorophyll contents of faba bean.This caused effect on chlorophyll levels enhancement in response to treatment with the seaweed extract, might be due to the presence of betaines. This suggestion could be reinforced by the conclusions of Whapham et al. (1993) and Gerald et al. (1997).

Moreover, growth criteria and chlorophyll contents of soybean plant showed highly significant increases as a result of algalization (Abdel Hadi et al., 1993 and Blunden et al., 1996).

Foliar fertilizers may work in part by being absorbed by roots when excess solution drips onto the soil. Davis (2004) emphasized the effect of these externally applied organic compounds, which could enhance plant growth.

\section{Conclusion}

The application of algal extract to improve crop yield and productivity could be recommended in the present work. Foliar sprays with algal extracts increased yield, seed index and quality of faba bean.

Most of the commercial seaweed extracts used across the world is usually prepared from the brown seaweeds (Fucales). In the present study, it was of interesting that the green algae (Halimeda opuntia and Ulva rigida) gave higher effects than Sargassum latifolium, which represented the used brown seaweed. This difference might emphasize the importance and necessity of future research dealing with biochemical screening and applications, using algal species belonging to different taxonomic groups collected from different ecological and geographical regions.

\section{Acknowledgment}

Acknowledgment is made to Dr. Osama Nagdi Massoud researcher in Agricultural Research Center (ARC) for the field experiment assistance, and N, P and $\mathrm{K}$ analysis of wheat plant.

\section{References}

Abdel Hadi, N. F.; Abou-Leila, B.; Emad, E. and Ahmed, K. H. (1993). The effect of three different types of soils on growth criteria, Rhotosynthetic pigments and yield of soybean fertilized with algae. Egypt. J. Appl. Sci., 8: 52-64. 
Beckett, R. P.; Mathegka, A. D. M. and Van Staden, J. (1994). Effects of seaweed concentrate on yield of nutrient-stressed tepary bean (Phaseoulus acutifolius Gray). J. Appl. Phycology. 6: 429-430.

Blunden, G.; Jenkins, T. and Liu, Y. W. (1996). Enhanced chlorophyll levels in plants treated with seaweed extract. J. Appl. Phycology. 8: 535-543.

Crouch, I. J. and van Staden, J. (1992). Effect of seaweed concentrate on the establishment and yield of greenhouse tomato plants. J. Appl. Phycology. 4: 291-296.

Davis, J. M. (2004). The integration of foliar applied seaweed and fish products into the fertility management of organically grown sweet peppers. Organic farming research Foundation (Final Project Report), 14p.

Dubois, M.; Gilles, K. A.; Hamilton, J. K.; Rebers, P. A. and Smith, F. (1956). Colorimetric method for determination of sugars and related substances. Analyt. Chem., 28: 350-356.

Duncan, D.B. (1955). Multiple range of multiple "F" tests. Biometrics, 11: 1-42.

El-Sheekh, M. M. and El-Saied, A . (2000). Effect of crude seaweed extracts on seed germination, seedling growth and some metabolic processes of Vicia faba L. Cytobios, 101:23-35.

Featonby-Smith, B. C. and van Staden, J. (1984). The effect of seaweed concentrate and fertilizer on growth and the endogenous cytokinins in Phaseolus vulgaris. S. Afr. J. Bot., 3: 375-379.

Gerald, B.; Teifryn, J. and Yan-Wen, L. (1997). Enhanced leaf chlorophyll levels in plants treated with seaweed extract. J. Appl. Phycology. 8: 535543.

Hu, X.; Jiang, X.; Hwang, H.; Liu, S. and Guan, H. (2004). Promotive effects of alginate-derived oligosaccharide on maize seed germination. J. Appl. Phycology. 16:73-76.

Løpez-Mosquera, M. E. and Pazos, P. (1997). Effect of seaweed on potato yield and soil chemistry. Biological Agriculture and Horticulture. 14:199-205.

Lowry, O.H.; Rosenbrough, N. J.; Farr A. L. and Randall R. J. (1951). Protein measurement with the folin phenol reagent. J. Biol. Chem., 193: 265275.

Malaguti, D.; Rombola, A. D.; Gerin, M.; Simoni, G.; Tagliavini, M. and Marangoni, B. (2002). Effect of seaweed extract-based leaf spray on the mineral status yield and fruit quality of apple. Acta Hort., 594:357-359.

Metzner, H.; Rau, H. and Senger, H. (1965). Untersuchungen, zür Synchronisierbarkei einzelner Pigment mangel Mutonaten von Chlorella. Planta. 65: 186-190. 
Mwanamwenge, J.; Loss, S. P.; Siddique, K. H. M. and Cocks, P. S. (1998). Growth, seed yield and water use of faba bean (Vicia faba L.) in a shortseason Mediterranean-type environment. Aust. J. Exp. Agric., 38: 171-180.

Norrie, J. and Hiltz, D. A. (1999). Seaweed extract research and applications in agriculture. Agro-Food-Industry Hi-Tech., March / April: 15-18.

Pirie, N.W. (1955). Modern methods of plant analysis. In: Peach, KK. And Tracey, M. V.) IV 23-68 Springer - Verlag, Berlin, Germany.

Temple, W. D. and Bomke, A. A. (1989). Effects of kelp (Macrocystis integrifolia and Ecklonia maxima) foliar applications on bean crop growth. Plant and Soil. 117: 85 - 92.

Wang, Q.; Shi, W.Y.; Rong, F. J.; Ma, J. W.; Guan, C. H. and Jiang, L. N. (2005). The effect of the liquid seaweed extract on resisting salinity stress of cucumber. Acta-Agriculturae-Zhejiangensis. 17: 268-272.

Whapham, C. A.; Blunden, G.; Jenkins, T. and Hankins S. D. (1993). Significance of betaines in the increased chlorophyll content of plants treated with seaweed extract. J. Appl. Phycology. 5: 231-234.

Williams, C. H. and Twine, J. R. (1960). Flame photometric method for sodium, potassium and calcium. In: Peachh, K. and Tracey, M. V. Modern methods of plant analysis Pp 3-5. Springer - Verlag, Berlin, Germany.

Woods, J. T. and Mellon, M. G. (1965). Chlorostannous - reduced molybdophosphoric blue colour method in sulfuric acid system, in: Jackson, M. L., Soil chemical analysis Prentice-Hall International Inc. London, UK, pp 141-144(1941).
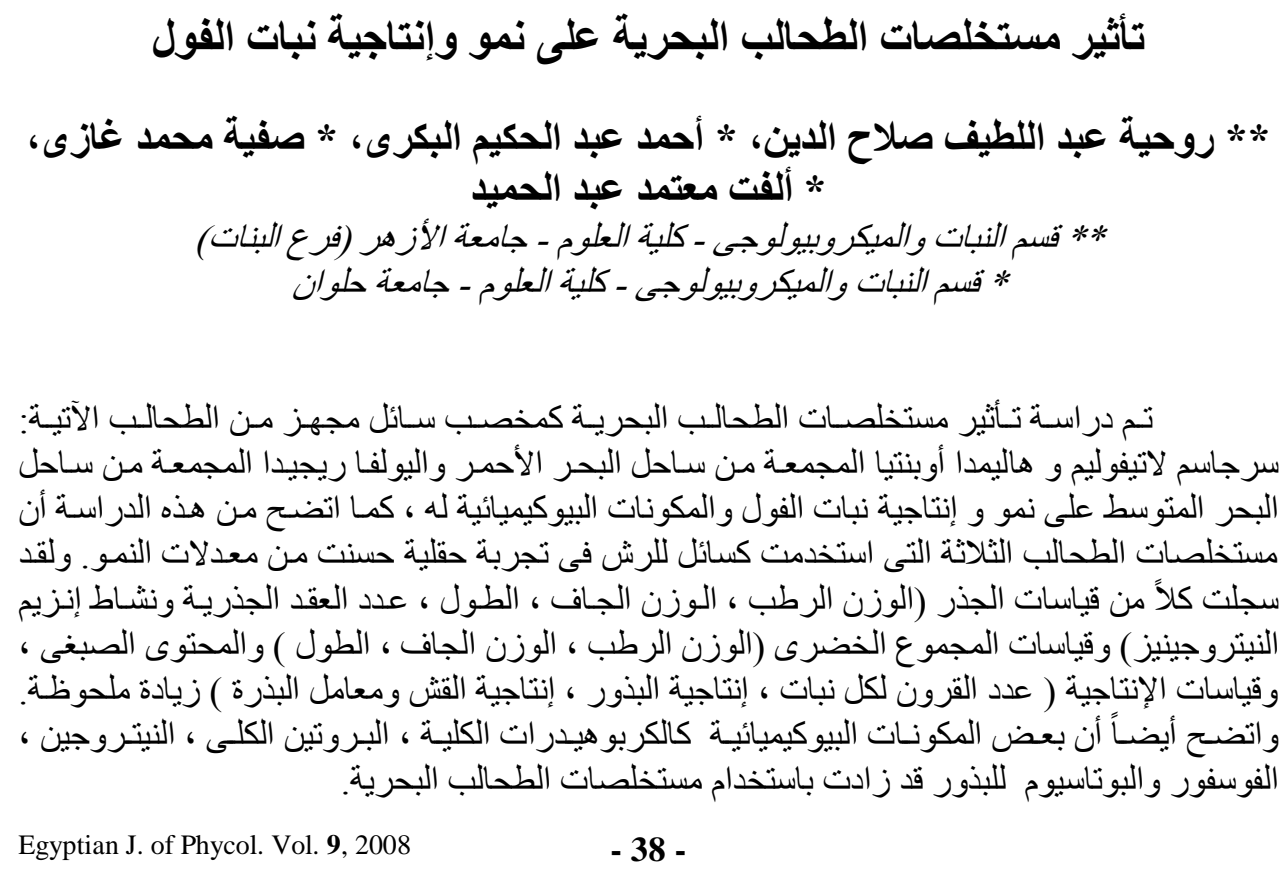